\title{
Laser-Scanning in vivo Confocal Microscopy of the Cornea: Imaging and Analysis Methods for Preclinical and Clinical Applications
}

\author{
Neil Lagali, Beatrice Bourghardt Peebo, \\ Johan Germundsson, Ulla Edén, Reza Danyali, \\ Marcus Rinaldo and Per Fagerholm \\ Additional information is available at the end of the chapter \\ http://dx.doi.org/10.5772/55216
}

\section{Introduction}

\subsection{The cornea is a model tissue for in vivo imaging}

The cornea is the most anteriorly located ocular tissue, serving principally as a transparent window for light to enter the human eye (Fig 1A). Besides the function of transparency, the curved cornea serves to refract light to deeper ocular structures, and presents a biological barrier to the outer environment. The cornea, often depicted histologically in cross-section, consists of five distinct layers: epithelium, Bowman's layer, stroma, Descemet's membrane, and endothelium (Fig 1B). For the interested reader, a detailed description of corneal anatomy and physiology can be found in Reference 1.

The cornea has long been considered to be a model tissue for microscopy, owing to the unique combination of various tissue elements (cells, extracellular matrix, nerves, vasculature, stem cells, etc.) present within a virtually transparent and relatively thin structure. The cornea transmits visible light with very little absorption, while the cellular features of interest within the cornea often strongly scatter light, resulting in the possibility of obtaining high signal-tonoise images by optical techniques. With the added advantages of confocal microscopy and the exterior anatomical location of the cornea, it has become a model tissue for high-contrast microscopic imaging in vivo.

\subsection{Endogenous-contrast darkfield confocal imaging of the living cornea}

Early confocal microscopy systems employed white light and tandem spinning discs to illuminate and collect light from a sample, while modern confocal systems use slit-scanning 

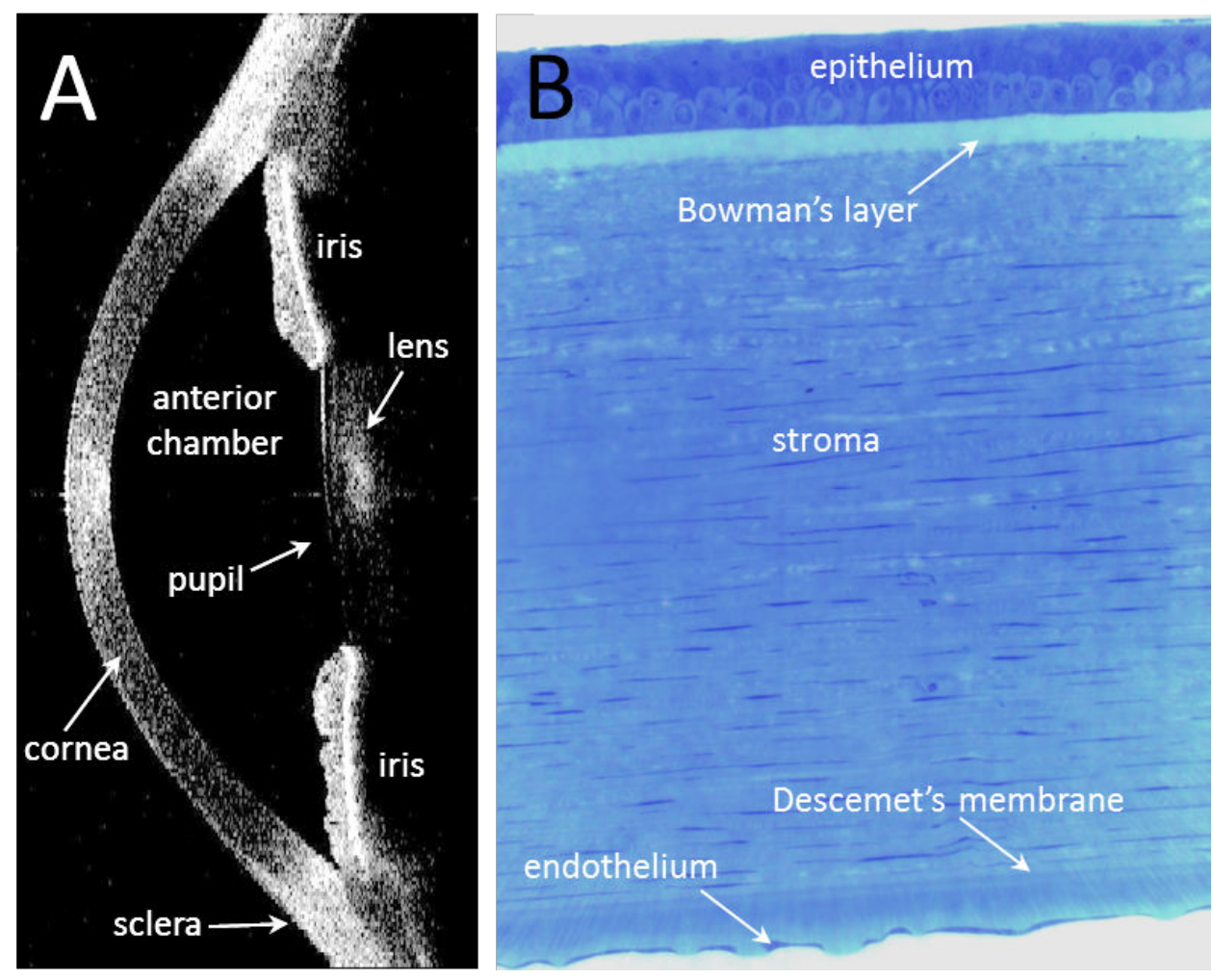

Figure 1. The human corneal anatomy. (A) In vivo cross-section of the cornea and anterior eye by anterior segment optical coherence tomography. The bright areas indicate light scatter while the dark areas are transparent. The bright area in the central cornea is an artifact. (B) Histologic view of the human cornea with toluidine blue staining, indicating the five principal layers of the normal cornea.

(white light) or point-scanning (laser) configurations. Regardless of the system, however, all confocal microscopes used to examine the cornea in vivo utilize the darkfield microscopy principle. In darkfield microscopy, only light scattered by the sample reaches the detector, while transmitted light is blocked, rendering transparent tissue or structures as 'dark'. Darkfield imaging has the advantage of achieving a high contrast of scattering structures against a dark background, and the potential for producing high-resolution images that approach or even exceed [2] the diffraction limit.

Another benefit is the endogenous contrast provided by structures in the tissue, which scatter visible light generally independent of wavelength and without the need for additional contrast-enhancing means. Practically, this endogenous contrast enables in vivo imaging of the inner structures of the cornea without the use of dyes, in a non-selective manner, since all scattering elements in the probed volume will be imaged, regardless of other biological properties. The clear advantage is that the tissue can be examined in vivo in its native state, with all cell types present. The challenge that then emerges, however, is to interpret in vivo 
images solely by morphology, without further cell-specific information. Some strategies for overcoming this limitation will be discussed later in this chapter.

\subsection{Equipment and procedures}

At present, there are two commercially-available systems for performing in vivo confocal imaging of the cornea. Both systems are manufactured for clinical use as medical devices. The Nidek Confoscan 4 from Nidek Technologies is a white-light, slit-scanning confocal microscope, while the Heidelberg Retinal Tomograph 3 with Rostock Cornea Module (HRT3-RCM) from Heidelberg Engineering is a red laser point-scanning confocal microscope (Fig 2A).
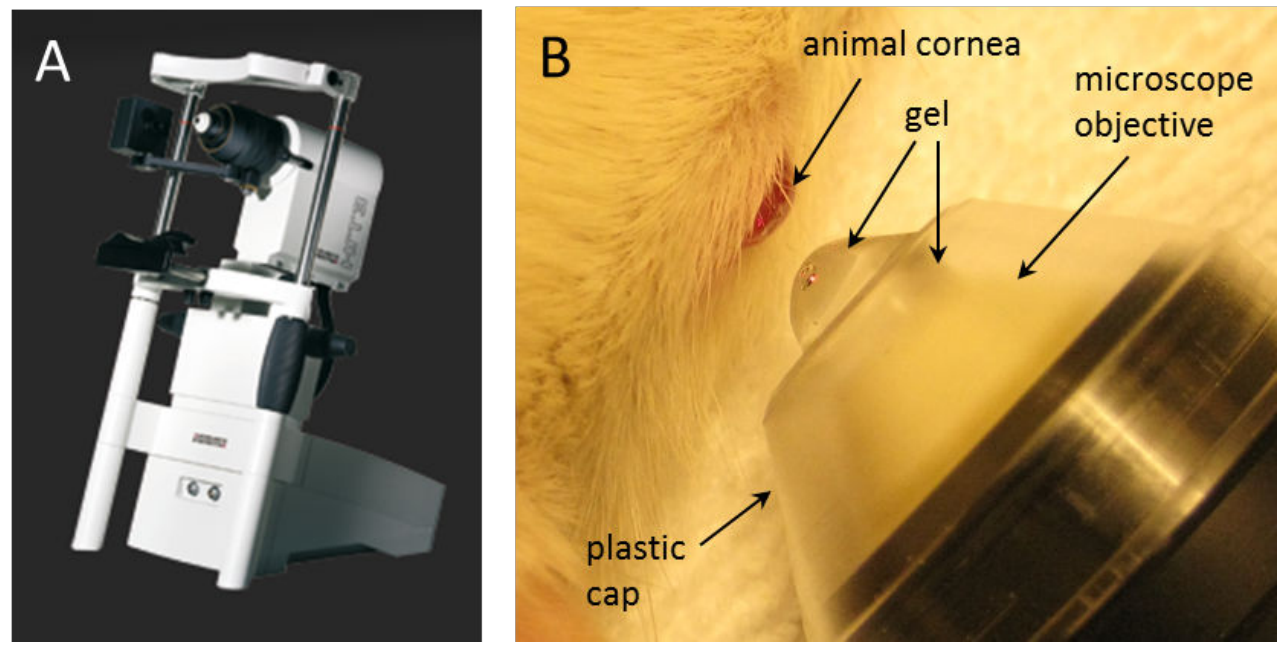

Figure 2. The HRT3-RCM system for laser-scanning in vivo confocal microscopy of the cornea. (A) The laser-scanning interface. (B) Magnified view of the scanning head with plastic cap and transparent tear gel applied. The gel and cap make contact with the ocular surface of the locally anesthetized animal (or human) cornea during imaging.

Consistent with the theme of this book, the remainder of this chapter will describe data obtained with the HRT3-RCM laser-scanning system. The system consists of a point-scanning $670 \mathrm{~nm}$ diode laser and horizontally-mounted optics to enable access to the cornea of a seated subject. The system and its operation are described in detail elsewhere. [3] The objective lens most commonly used is a water immersion lens with high numerical aperture (63x/0.95 NA, Zeiss), upon which a disposable sterile plastic cap is placed. The cap comes into physical contact with the corneal surface through a refractive index-matching ophthalmic gel (eg., ViscoTears, Novartis). An in vivo confocal microscopy (IVCM) examination consists of one or several scans, where each scan consists of 100 consecutive images acquired at a selectable rate from 1 to 30 frames/sec. During the scan (which lasts from 3.3 to $100 \mathrm{sec}$, depending on the acquisition rate), the operator manually adjusts the position of the objective relative to the cornea in a plane parallel to the corneal surface. Additionally, the operator adjusts the depth of focus in the axial direction, by manual or motor-driven [4] means. A depth range of 
approximately $1200 \mu \mathrm{m}$ is available, which is ample for examination of human corneas typically $550 \mu \mathrm{m}$ thick. In this manner, the entire corneal volume can be accessed for in vivo imaging. Practically, access to corneal regions further from the apex is achieved by manually placing a movable fixation target into which the patient is instructed to view.

The images acquired represent a field of view of either $300 \times 300 \mu \mathrm{m}$ or $400 \times 400 \mu \mathrm{m}$, depending on the choice of internal field lens (which is user-interchangeable). The lateral resolution is about 1-2 $\mu \mathrm{m}$, but can be submicron depending on contrast [2], while the axial resolution is about $4 \mu \mathrm{m}$. [5] Comparable figures for the slit-scanning microscope are 1-2 $\mu \mathrm{m}$ lateral and 25-27 $\mu \mathrm{m}$ axial resolution. [6,7]

Patient examinations in the clinic are conducted upon a topically anesthetized cornea, and depending on the clinical and/or scientific questions, a number of scans are completed during a given session. Upwards of 20 scans per eye (2000 single images per eye) is not uncommon. Typical examinations may last from 5 to 15 minutes, with patients rarely experiencing discomfort. For patients, an image acquisition rate of 8 frames/sec is typical, however in certain cases (eg., a subject with nystagmus) the frame rate is increased.

For preclinical animal imaging, the animal is examined under general anesthesia and with topically applied anesthetic drops. Animals are examined in the prone position, while resting on a movable stand (Figure 2B). During examination, care is taken to keep the cornea hydrated with saline drops. Image acquisition is similar as for patients, but typically with a slower frame rate ( 5 frames/sec) due to the minimal motion of the animal during examination. Often anatomic considerations limit the accessible region of the animal cornea. In order to obtain good in vivo images, the animal must be oriented such that the corneal surface is presented tangentially to the microscope objective lens. This often requires manual manipulation of the head and eyelids, which in practice means at least two persons are needed to conduct the examination.

\subsection{Application of IVCM to clinical and animal research}

The use of IVCM in scientific research in ophthalmology has rapidly expanded over the past decade. The interested reader is referred to several informative review articles describing the use of IVCM in humans, [5], [8]- [10] however, reviews are barely keeping pace with the latest developments in the field. The use of IVCM in preclinical animal research is more recent, and is slowly gaining momentum. Animal studies have detailed the animal corneal anatomy [11], the effect of various surgical techniques, [12]- [14] and phenomena such as inflammation [15] and angiogenesis. [16], [17]

Besides research, IVCM has been used for clinical diagnosis of pathology of the cornea, or as a screening/monitoring tool for patients undergoing treatment. [18]- [22] The number and scope of clinical applications of IVCM will undoubtedly increase in the coming years.

It is pertinent to note that ethical considerations in human and animal research can be influenced by the use of IVCM. In human studies, the principle of informed consent is relevant, and the non-invasive nature and short-duration of IVCM examination can facilitate patient and ethical review board acceptance. It must also be noted that the laser is non-damaging to 
the eye, and the instrumentation is approved for clinical use in humans. In animal research, IVCM facilitates repeated, longitudinal examination of the same animal corneas over periods of hours, days, months, or years. [12], [13], [16] While the technique can therefore reduce the number of animals required for a study (since repeated non-invasive cellular-level examination is possible in the same animal), care must be taken to administer anesthesia in a humane manner. Typically, for small animals such as mice or rats, short-duration or time-lapse studies can be performed by repeated general anesthesia over a period of several hours. It is not advisable for animals to remain under general anesthesia for longer time periods, and it is advised to administer an antidote (such as atipamezole) where feasible, to rapidly reverse the effects of the anesthetic.

\section{Feature recognition in IVCM}

\subsection{IVCM appearance of the normal human cornea}

A summary of the different layers and features of the normal human cornea is presented in Figure 3 . The different layers are imaged by adjusting the axial alignment to select a given focal depth in the cornea.
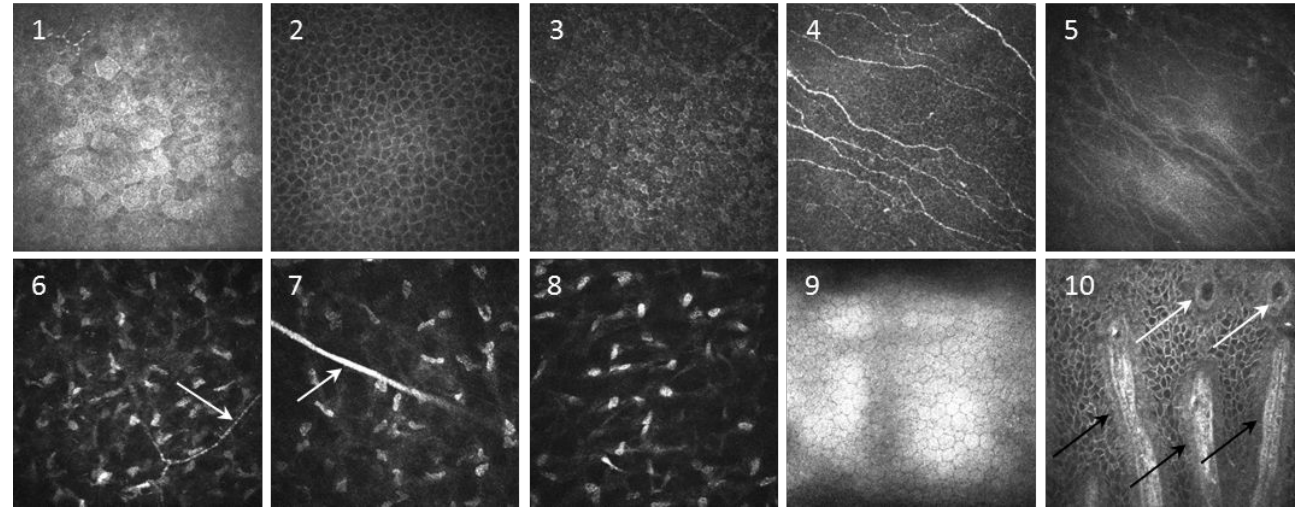
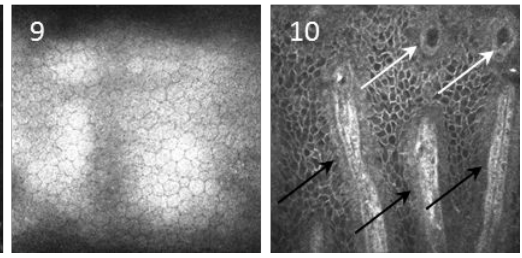

Figure 3. IVCM appearance of the corneal layers in a normal human subject. Depths from the corneal surface are given. All images are $400 \times 400 \mu \mathrm{m}$. 1. Depth $0 \mu \mathrm{m}$, superficial epithelium; 2. Depth $20 \mu \mathrm{m}$, epithelial wing cell layer; 3 . Depth $30 \mu \mathrm{m}$, basal epithelium; 4. Depth $40 \mu \mathrm{m}$, subbasal nerve plexus; 5. Depth $45 \mu \mathrm{m}$, Bowman's layer; 6 . Depth $150 \mu \mathrm{m}$, anterior stroma with nerve (arrow); 7. Depth $200 \mu \mathrm{m}$, mid stroma with nerve trunk (arrow); 8. Depth $400 \mu \mathrm{m}$, posterior stroma; 9. Depth $530 \mu \mathrm{m}$, endothelium; 10. Inferior limbal palisade ridges (black arrows) and focal stromal projections (round protrusions, white arrows) indicating the presumed limbal epithelial stem cell niche.

The en face images parallel to the corneal surface shown in Figure 3 are the most widely used; however, the instrument offers the possibility of oblique imaging when the microscope objective contacts the cornea off-axis. Although the scale of the image is difficult to ascertain in oblique images, the information yielded by such images is useful for localizing structures or pathology within the cornrea in a picture similar to a histological cross section (Figure 4). 


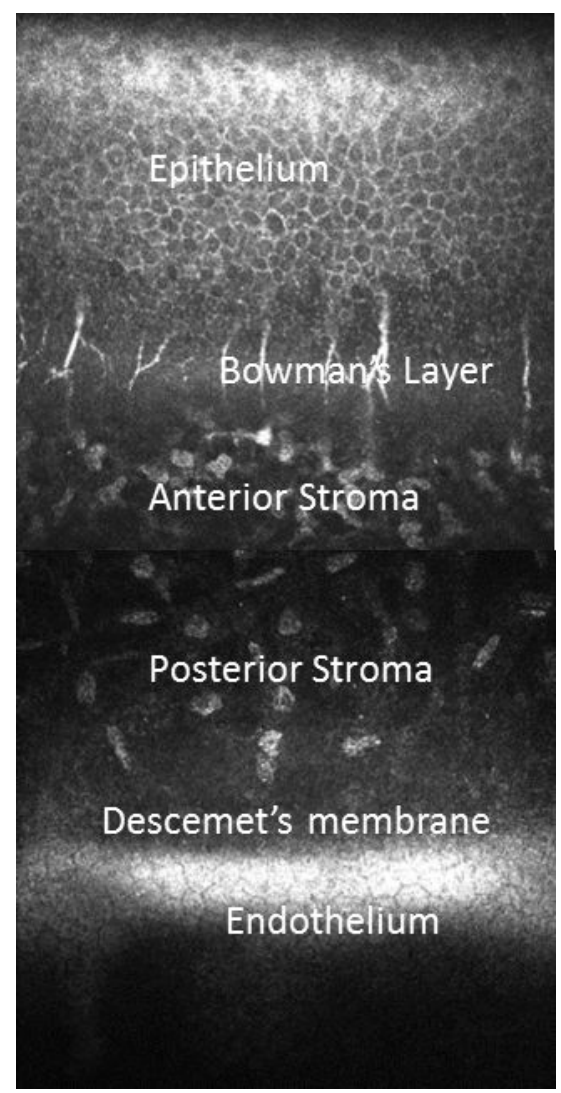

Figure 4. Live histological cross-section representation of a normal cornea by IVCM. Compare this in vivo view with that of the cross-section in Fig 1B.

\subsection{Application: Distinguishing different inflammatory cell subtypes in corneal inflammation}

IVCM can be used to identify the presence of inflammatory cells in the cornea, despite their invisibility by traditional methods of clinical observation such as slit lamp biomicroscopy. Moreover, the morphological appearance of the various inflammatory cell types in vivo enables the distinction between certain cell subtypes including neutrophil-granulocyte, immature/mature dendritic, and macrophage (Figure 5). Although these cells of leukocytic origin can invade the cornea from the peripheral limbus and conjunctiva in cases of overt, active inflammation, it is also important to note that IVCM can detect early inflammatory cell influx prior to the appearance of external signs of inflammation. Additionally, inflammatory cell presence can be detected in vivo in cases of chronic, sub-clinical inflammation as observed in some corneal transplant patients (Figure 5). Dendritic cells in the cornea have been the most widely studied inflammatory cell subtype by IVCM. While populations of dendritic cells are 
resident in the peripheral human cornea under normal conditions, [23] in an inflammatory setting these cells migrate to the central cornea and mature into MHC class $\mathrm{II}^{+}$cells capable of antigen presentation. [24]
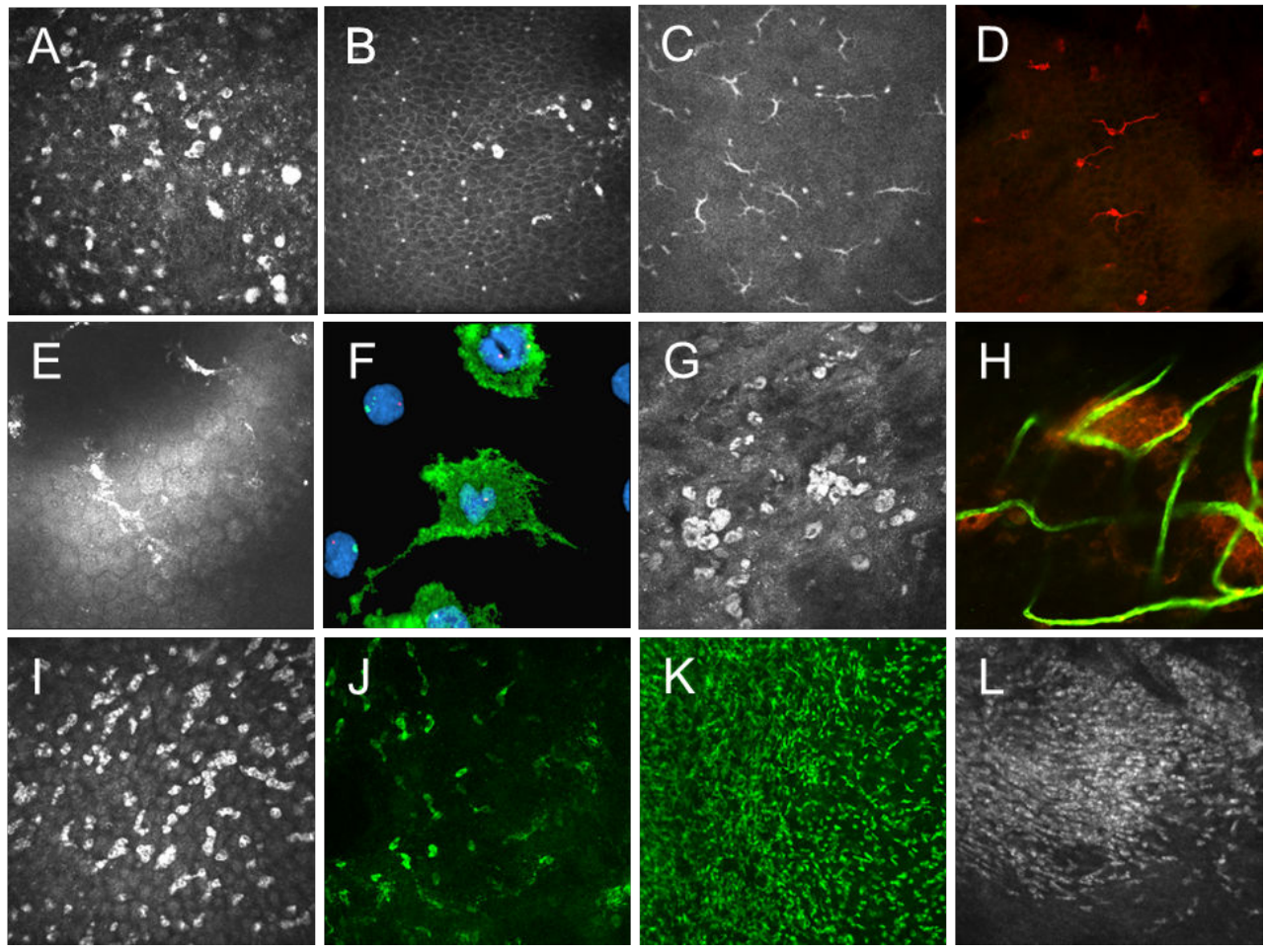

Figure 5. Inflammatory cell imaging by IVCM. (A-B) Leukocyte invasion of the epithelium one year after corneal transplantation in two patients. (C) Dendritic cells with long dendrites and dendritic cell bodies lacking dendrites in the central cornea 2 years after transplantation. (D) CD45+ dendritic cells (red) in the rat corneal epithelium. (E) Inflammatory cells on the endothelial surface. (F) CD45+ leukocytes (green) on the endothelial surface of a failed human transplant, with DAPI-counterstained nuclei (blue). (G) Mature macrophages in a patient with regressed neovessels. (H) $\mathrm{KiM}_{2} \mathrm{R}^{+}$mature rat macrophages (red) during neovessel regression (CD31+ vessels, green). (I) Neutrophil-granulocytes invading the corneal epithelium in a patient. (J) CD11 b+ neutrophil-granulocytes (green) in the rat. (K) Massive influx of CD11b+ myeloid cells (green) from the conjunctiva into the corneal stroma in acute inflammation in the rat. (L) In vivo image of the myeloid cell influx during inflammation in the rat.

\subsection{Feature recognition for diagnosis - Application to acanthamoeba keratitis}

Acanthamoeba keratitis (AK) is a serious, sight-threatening infection of the cornea by the acanthamoeba parasite. [25] The parasite first establishes itself as an amoeboid body (trophozoite) on the ocular surface, where it gradually kills epithelial cells by phagocytosis or cell lysis. As it matures, the parasite forms characteristic double-walled cysts, varying in size from 5 $200 \mu \mathrm{m}$ in diameter typically. If left untreated, the parasite will continue to migrate through to 
the stroma, where it induces inflammation, scarring, and eventually corneal ulceration and perforation, leading to blindness. AK can be difficult to diagnose owing to the relative insensitivity of current biosampling and culture methods; however, its timely diagnosis is paramount for administering the correct treatment and for optimal clinical outcomes.

In recent years, IVCM has become an important tool for screening and diagnosing patients suspected of having AK. The interested reader is referred to recent literature on the subject of AK diagnosis by IVCM. [26]- [28] Several unresolved issues remain, however, with this in vivo diagnostic technique. Sensitivity and specificity of diagnosis are instrument and operatordependent, [26], [27] and the in vivo images are often difficult to interpret and no clear guidelines for interpretation exist. Guidelines for performing IVCM examination in suspected cases of AK are likewise lacking.

Generally, exams should include focusing on superficial epithelial layers and performing wide-area scans across the ocular surface, using the fixation target to access peripheral zones. Attention should especially be given to zones where the epithelium exhibits defects. Most cysts detected in the clinic are present in the superficial epithelial layers. Repeated examination sessions on the same day or on consecutive days are recommended, particularly in suspected cases where no positive confocal evidence is found initially.

Several in vivo images from patients with suspected AK are shown in Figure 6. It is apparent that the morphologic appearance in terms of size, reflectivity, density of cells, and presence of the double-wall in cysts can vary widely across patients. Upon comparing Figures 5 and 6, it can be seen that cysts can be easily misinterpreted as inflammatory cells and vice-versa. It is also of note that images obtained with white-light slit-scanning confocal systems [26] are markedly more difficult to interpret than those obtained with the laser-scanning system, due to the increased axial resolution of the latter.

\subsection{Keratocyte apoptosis}

Keratocytes normally appear in IVCM examinations as bright nuclei with transparent or semitransparent cell bodies in the normal cornea (panels 6-8 in Figure 3). Keratocyte death by apoptosis occurs in the cornea in cases of stromal perturbation such as external trauma, or with aging in normal subjects. Comparing the stromal keratocytes in healthy younger and older subjects, a lower density of keratocytes is observed [29] and the presence of a large number of fine 'needle-like' structures is evident in older corrneas (Figure 7).

Needle-like structures are also observed in the transplanted cornea, concomitant with a sparse presence of keratocytes. The structures observed at various stages in the apoptotic process include a pronounced increase in reflectivity of the keratocyte body, elongation of the nucleus and cell body into a linear form, condensation of this linear form into a thin, needle-like structure, and the presence of condensed, punctate inclusions of smaller size and reduced reflectivity relative to the healthy keratocyte nucleus (Figure 8).

In keratoconus patients treated with UVA-riboflavin corneal collagen cross-linking (CXL), cells in the ultraviolet-irradiated region of the cornea undergo apoptosis en masse, resulting in a dramaticchangein the cornealstromalappearance, withkeratocytesinitially absentin thetreated region. In border regions of the stroma, needles are observed (Figure 8). The features observed 

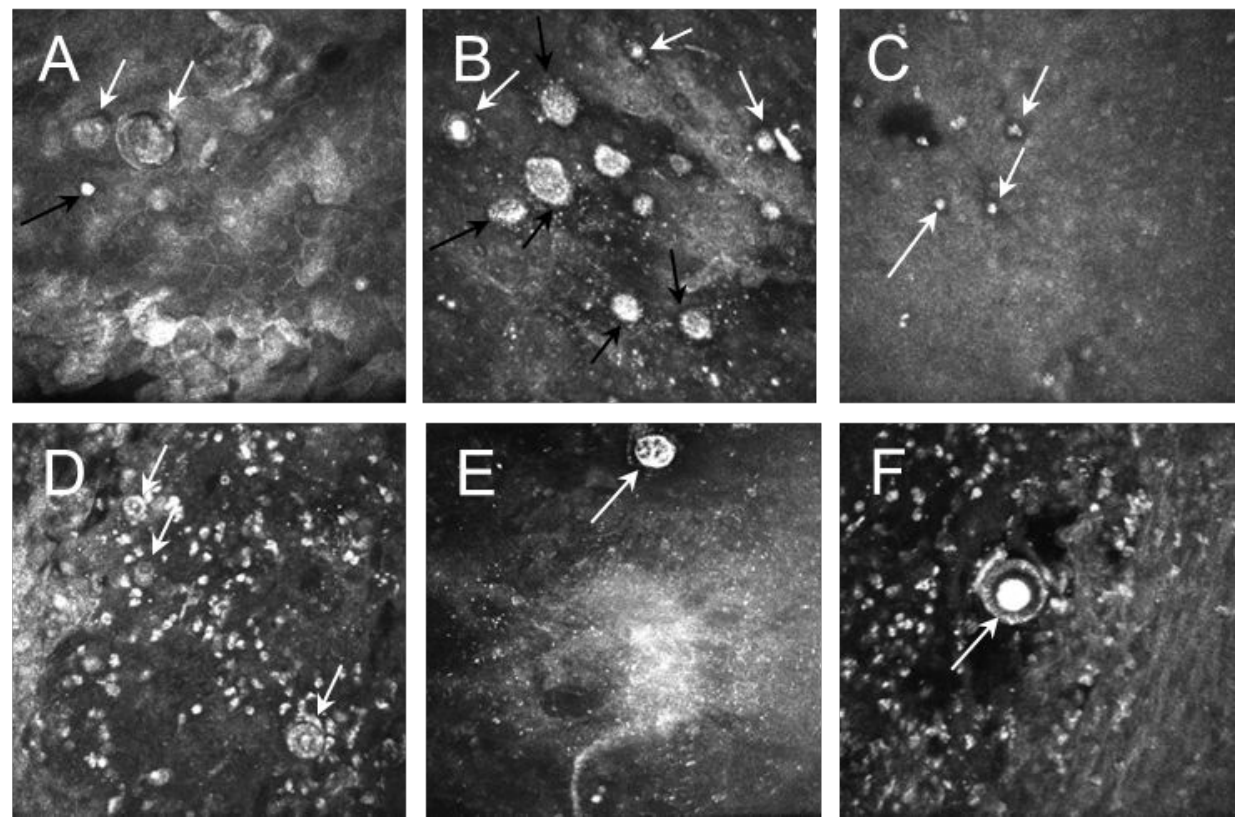

Figure 6. IVCM images in several suspected cases of AK. (A) Superficial epithelial double-walled cysts (white arrows) and suspected early-stage cyst (black arrow). (B) Acanthamoeba cysts of varying size (white arrows) and suspected trophozoites (black arrows). (C) Suspected early-stage cysts (arrows) appear similar in morphology to inflammatory cells, however, the latter generally appear in greater numbers. (D) Inflammatory cells and double-walled cysts (arrows). (E) a large cyst (arrow) in a region devoid of epithelial cells. (F) Large cyst with clear double-wall morphology (arrow).

in patients treated by CXL are mimicked in a rat model, where IVCM images indicate the same structures observed in humans, with immunohistochemical staining confirming apoptosis (Figure 8). The length of time apoptotic remnants persist in the corneal stroma is unknown; however, their continued appearance months or years after trauma suggests a slow turnover.

\subsection{Detection of the limbal epithelial stem cell niche}

An emerging clinical application for IVCM is in the assessment of the limbal epithelial stem cell niche in patients with limbal stem cell deficiency. A few studies have described the in vivo morphological characteristics of the limbal palisades region of the corneal epithelium, believed to harbor limbal epithelial stem cells. [30]- [33] The structures associated with the stem cell niche are the palisade ridges and focal stromal projections at the basal limbal epithelium, and cells positive for putative stem cell markers reside at the base of these structures. [31] In Figure 9, these structures are depicted in normal healthy subjects, where palisade ridge morphology and the distribution of focal stromal projections is seen to vary. The structures additionally have a morphologic appearance that varies with skin pigmentation of the subject [30], [33] which is an important consideration when assessing the palisade morphology. 

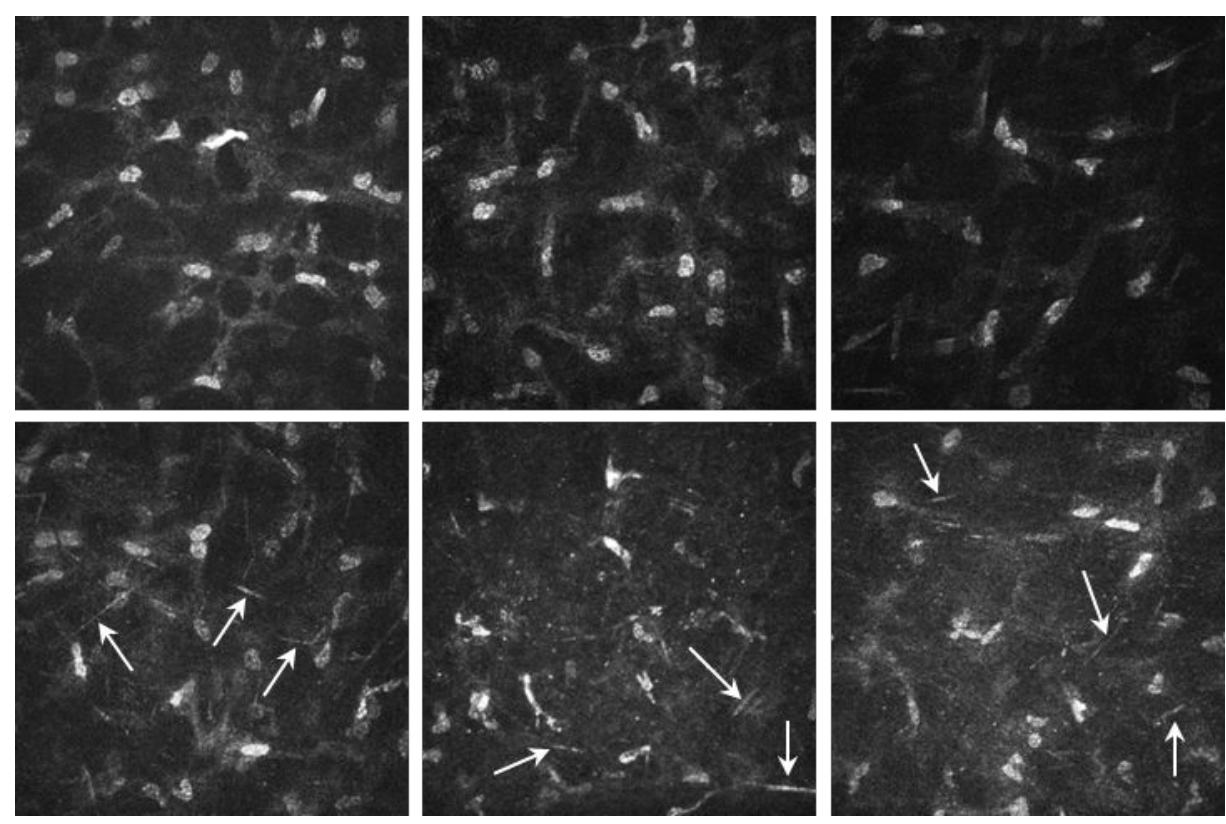

Figure 7. Normal healthy corneas at mid-stromal depth. Top row, young subjects $(23,21,16$ years of age, from left to right). Bottom row, older subjects (88, 77, 76 years) exhibit fine, reflective needle-like structures (arrows) indicating keratocyte apoptosis, not generally present in the younger corneas.

IVCM can also assist in assessing the degree of limbal stem cell deficiency in a patient. [34]- [36] In practice, the limbus of a patient is imaged at nasal, temporal, inferior and superior regions to document the limbal palisades. Most often, a superior and inferior examination suffices, as stem cells are predominantly located in these regions. In cases of limbal stem cell deficiency, the palisades may appear degraded or absent, replaced with blood vessels, inflammatory cells, and highly scattering conjunctival tissue (Figure 9).

With further detailed studies of normal and stem cell deficient corneas, IVCM could become an important tool in assessing degree of trauma, risk for surgical intervention, and prognosis in treated or stem cell transplanted patients.

\section{Nerve analysis and quantification methods}

\subsection{Corneal nerve anatomy}

The cornea is one of the most densely innervated tissues in the body. Nerves enter the cornea from a peripheral, mid-stromal depth as nerve trunks, then branch as they proceed anteriorly to eventually terminate at single nerve terminals between corneal epithelial cells at the ocular surface. For an in-depth discussion of corneal nerves and their detailed anatomy, the reader 

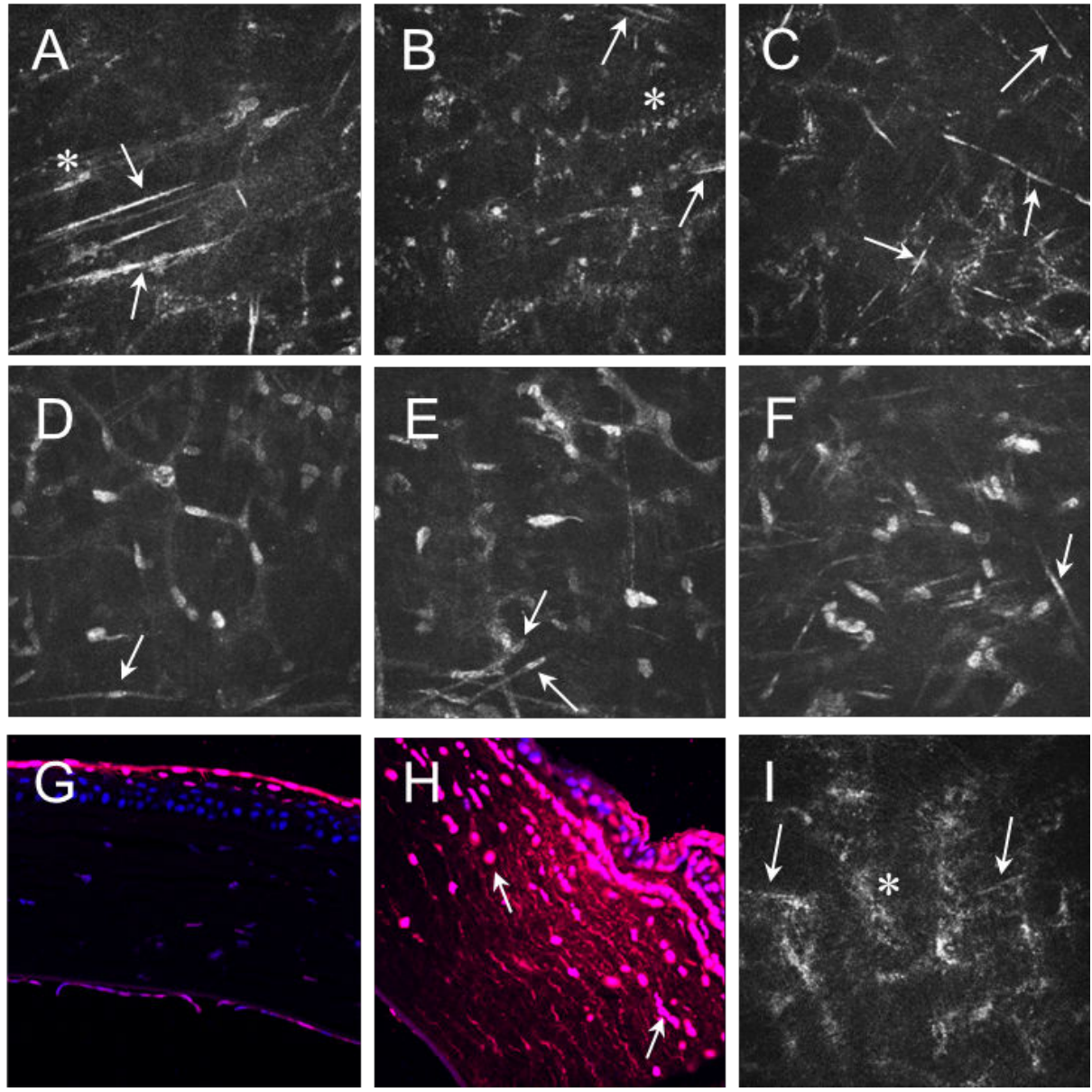

Figure 8. Morphologic appearance of apoptotic keratocytes. Top row: stroma of the transplanted cornea, middle row: stroma adjacent to a collagen-crosslinked region, bottom row, images from a rat cornea treated by the CXL procedure. (A) Prominent needles (arrows) and an elongating keratocyte (asterisk) in a patient 2 years after corneal transplantation. Note the scarcity of keratocytes. (B) A stromal region devoid of keratocytes, with needles (arrows) and condensed bodies (asterisk) visible in a patient 2 years after transplantation. (C) Needles (arrows) in a region of stroma devoid of keratocytes 4 years after transplantation. (D-F) Keratocyte elongation (arrows) during apoptosis in a 27-year old patient 4 months after receiving CXL treatment for keratoconus. (G) Immunofluorescent section from a normal, unaltered rat cornea marked with apoptotic marker BrdU (red) and nuclear counterstain 7-AAD (blue). Note the apoptosis in the superficial epithelium. (H) Section from a rat 1 day after $\mathrm{CXL}$ treatment, indicating significant anterior keratocyte apoptosis (arrows). (I) The same rat cornea at 1 day in vivo exhibited a loss of keratocytes, needles (arrows) and condensed bodies (asterisk).

is referred to several recent publications. [37]- [39] The subbasal nerve plexus in particular, located at the border between Bowman's layer and the basal epithelium, has become a landmark within the cornea owing to the concentration of nerves at this plane and the ease of imaging this layer with high contrast by confocal microscopy. The subbasal nerves gently 

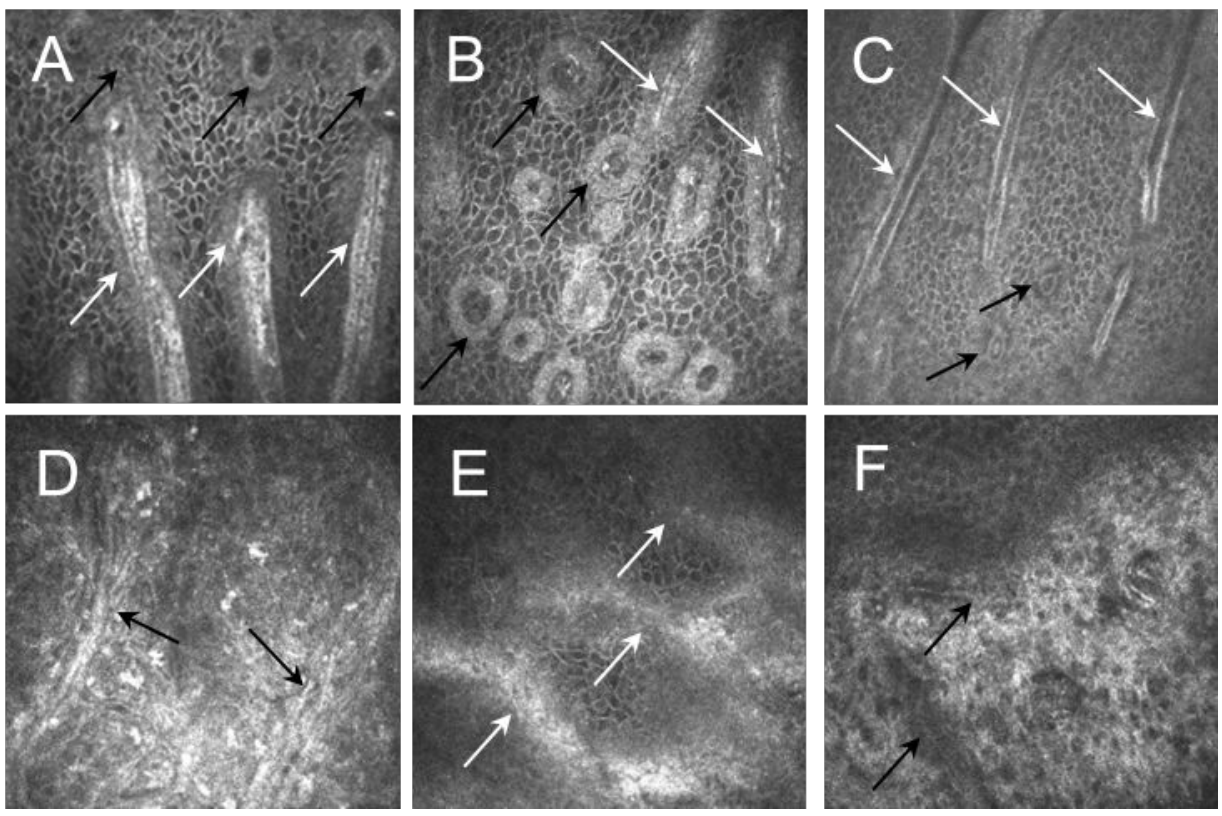

Figure 9. Limbal epithelial stem cell assessment in vivo. (A-C) appearance of the limbal palisades in normal healthy subjects. (D-F) appearance of the palisades in stem cell deficient patients with aniridia. (A-C) Palisade ridges (white arrows) and focal stromal projections (black arrows) exhibit varied diameter, cell appearance and distribution. (D,E) Degraded remnants of palisade ridges are visible (arrows). (F) Total absence of the stem cell niche in a conjunctivalized limbus, where blood vessels (arrows) are apparent amidst the highly scattering conjunctival tissue.

spiral inwards from the periphery to the corneal apex (Figure 10), and may follow the pattern of epithelial cells as they regenerate. [36], [40] Notably, the subbasal nerves have been shown to be perturbed in many pathologies and in cases of trauma. [38], [41] An example of a pathologic subbasal nerve architecture in keratoconus is depicted in Figure 10.

\subsection{Nerve analysis techniques}

Nerve analysis can be aided by qualitative and quantitative processing techniques. Nerve montages such as that depicted in Figure 10 can aid in qualitative assessment of nerve morphology. Recently, several authors have proposed various techniques to automate the process of creating nerve montages from the individual IVCM images obtained by adjusting fixation during patient examination. [42], [43] The quality of the resulting montage, however, is limited by the examination procedure. Care must be taken such that a minimum pressure is applied on the cornea by the plastic microscope objective cap during examination - otherwise, unwanted striations will occur and reduce the image quality. [44]

Quantitative nerve analysis has been performed by a number of investigators [36], [38], [39], [41] and in the case of the subbasal nerve plexus, reporting of nerve density has followed an accepted convention. Nerve density values determined from laser-scanning confocal instru- 

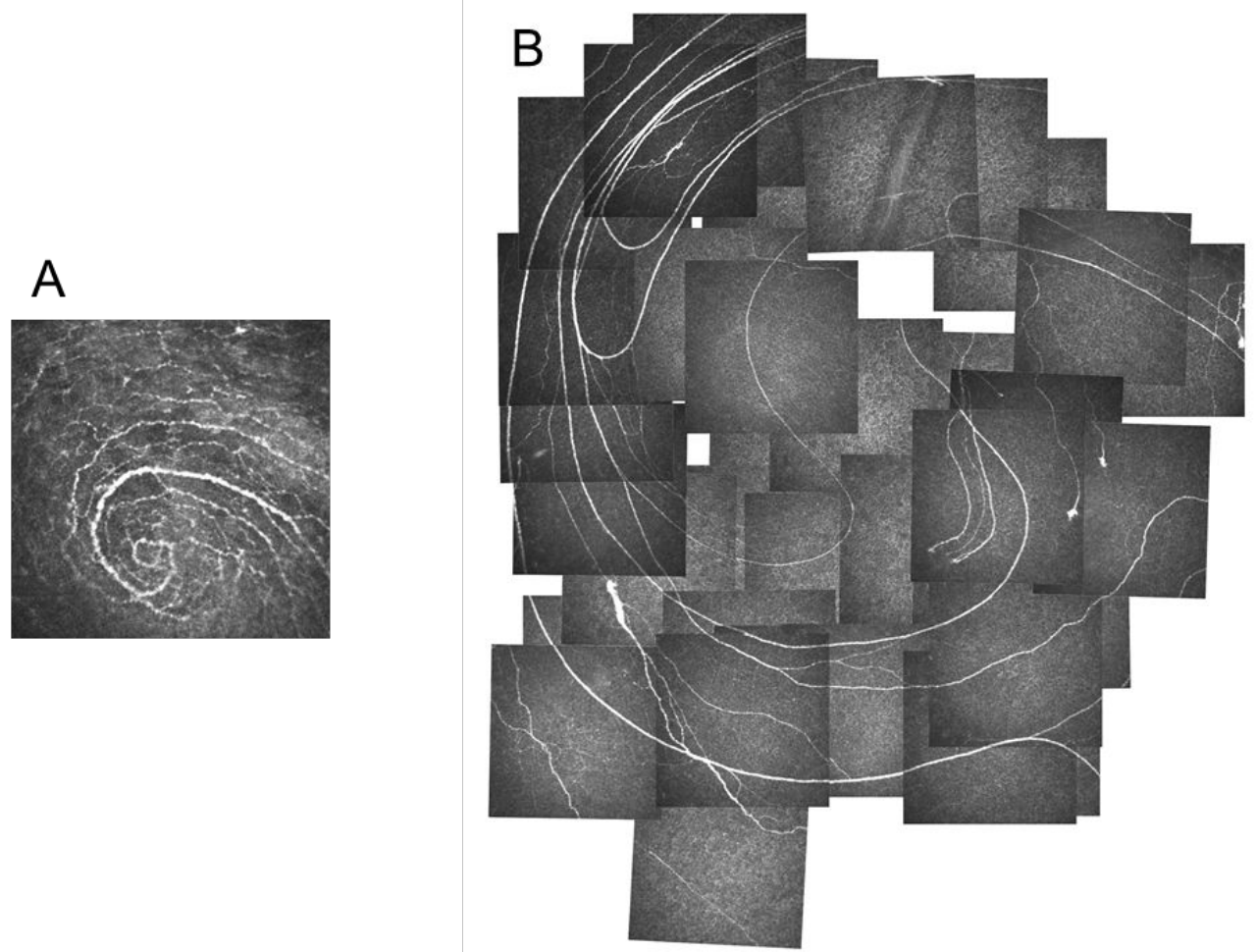

Figure 10. Human corneal subbasal nerve architecture. (A) A prominent whorl of subbasal nerves into the infero-central corneal apical region. (B) The whorl pattern is missing in this montage of the subbasal nerve plexus in a keratoconic cornea. Instead nerves, form characteristic looping structures.

ments can therefore be compared. Nerve density is reported in terms of total nerve length per unit area of the central corneal subbasal nerve plexus. In practice, a single $400 \times 400 \mu \mathrm{m}$ field of view image obtained from the central cornea is chosen, typically with the greatest number of visible subbasal nerves. Nerves in the image are then semi-automatically traced by the aid of tracing software [12], [19], [36] to determine total nerve length, which is then divided by the area in the field of view, to determine nerve density in $\mu \mathrm{m} / \mathrm{mm}^{2}$. The subbasal nerve density in humans changes with age, [29] but in general the accepted value for the average density by IVCM is about $20,000 \mu \mathrm{m} / \mathrm{mm}^{2}$ in the central cornea. [10], [29], [38], [39] Nerve density measurement according to this technique, however, is dependent upon where the single image is sampled. Images from the whorl region typically have the highest density while those in the paracentral region may have significantly reduced density. [39] Moreover, the clarity and contrast of the nerve image may render more nerves visible (including secondary connecting branches), while images with too much mechanical pressure placed on the cornea may disrupt the nerve pattern. Scattering from the epithelial basement membrane and/or anterior Bowman's layer can also influence visibility of the subbasal nerves (Figure 11). Therefore, wide- 
area montages such as that shown in Figure 10 are recommended for quantification of the average central subbasal nerve density, with the recognition that a qualitative picture of nerve architecture may be equally useful. While some parameters such as nerve branching and tortuosity have been quantified by some investigators, consensus on analysis techniques of the subbasal nerve architecture is still required. Moreover, the subbasal nerve density as observed by IVCM likely excludes most of the interconnecting nerve fibers between the larger subbasal nerve bundles. [45], [46] With these bundles included, the average subbasal nerve density is upwards of $50,000 \mu \mathrm{m} / \mathrm{mm}^{2}$ in the central cornea. [45]
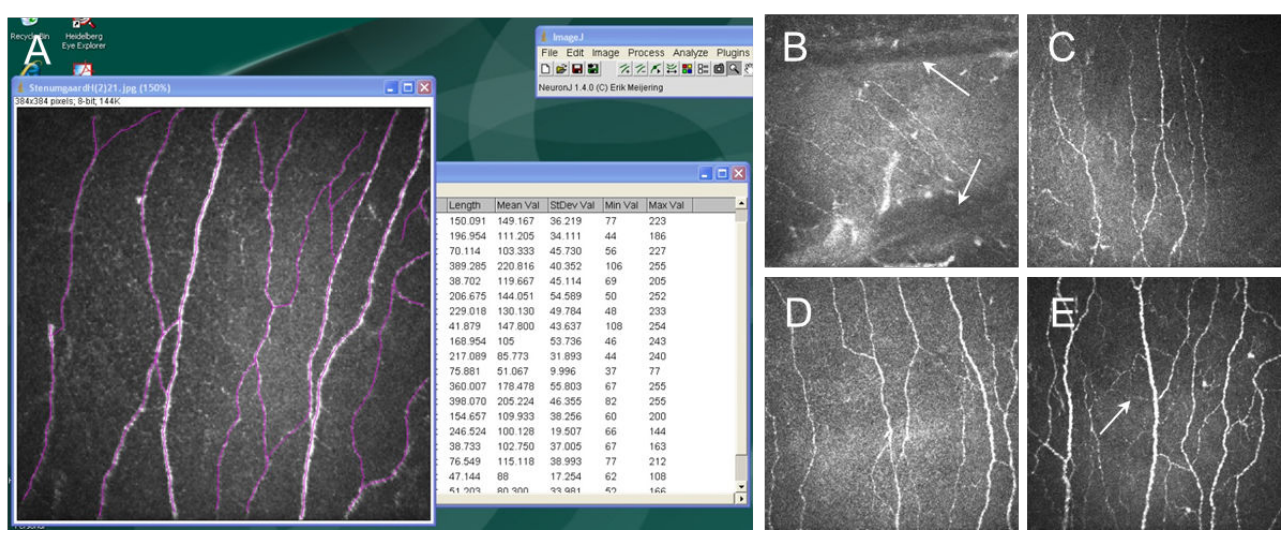

Figure 11. Subbasal nerve density analysis with IVCM. (A) An example of software-aided nerve tracing and quantification of nerve length. (B-E) Images taken from the central subbasal nerve plexus in healthy volunteer subjects. (B) Artifacts (arrows) caused by mechanical pressure on the cornea. These reduce the effective area in which nerves can be quantified, resulting in an artificially low nerve density. $80 \mathrm{y}$ old male subject, density $10,000 \mu \mathrm{m} / \mathrm{mm}^{2}$. (C) A slightly oblique section with different corneal depths apparent in the same image, causing nerve density to be underestimated. 35 year old male, density $26,000 \mu \mathrm{m} / \mathrm{mm}^{2}$. (D) Subbasal nerves with basal epithelial cells of increased reflectivity rendering few interconnecting nerve branches visible. 23 year old male, density $24,500 \mu \mathrm{m} / \mathrm{mm}^{2}$. (E) Nerve plexus with good contrast and low basal cell reflectivity enables interconnecting nerve branches to be seen. 55 year old male, density $33,600 \mu \mathrm{m} / \mathrm{mm}^{2}$.

\subsection{Assessment of nerve density in corneal epithelial basement membrane dystrophy}

In the cornea, epithelial basement membrane dystrophy (EBMD) is one of the most common diseases that can threaten vision. In EBMD, adhesion between the epithelium and the underlying Bowman's layer is defective. This disrupted epithelial barrier leads to abnormal epithelial cell and basement membrane physiology that can result in reduced vision and painful erosions of the epithelial cells. [19], [47]

As the disease affects the region where the subbasal nerve plexus is located, it is desirable to know whether the nerves are also affected. Central subbasal nerve density in corneas with EBMD (virgin eyes where no surgical treatment has been applied) can be determined by IVCM and nerve tracing techniques, and compared to the nerve density in a control group of healthy individuals. Comparing nerve density in 40 healthy control subjects with that of 24 corneas 
from 24 patients with EBMD, it is clear that the subbasal nerve density is reduced in EBMD (Figure 12).

The analysis was achieved by first selecting good quality subbasal nerve images without artifacts, then coding images randomly, such that in further analysis the analyzer is masked to the origin of each image. The coded images are then subjected to nerve tracing by two trained independent observers, ideally on the same computer monitor on separate occasions. The results are then unmasked and the average density per image is taken. In addition, a BlandAltman analysis can be performed to indicate the overall level of agreement in nerve density between observers. [19]
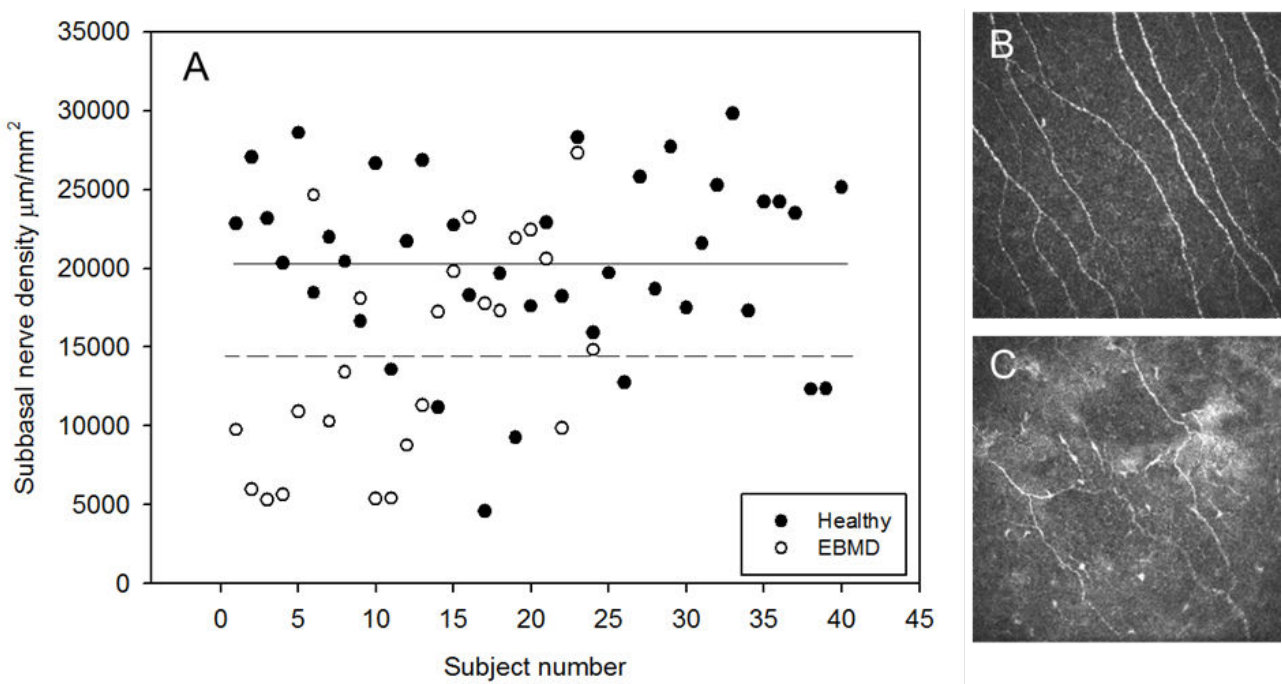

Figure 12. Assessment of nerve density in EBMD patients and healthy subjects. (A) Nerve density in EBMD patients (open circles) is on average $14,000 \mu \mathrm{m} / \mathrm{mm}^{2}$ (dashed line), which is below the average level of $20,000 \mu \mathrm{m} / \mathrm{mm}^{2}$ in healthy subjects (solid line). This difference is visible upon comparing images of a healthy plexus (B) with that obtained from an EBMD patient (C).

\subsection{Longitudinal quantification of nerve regeneration in corneal transplants}

Repeated, longitudinal quantification of nerve density can aid in the determination of nerve recovery after trauma, pharmacological treatment, or surgical intervention. Nerves can thereby serve as an indicator of wound healing and restoration of epithelium and ocular surface integrity. It is recommended, where possible, to correlate nerve presence with additional testing of nerve function by esthesiometry.

In the following example, the same group of 9 transplant patients was examined by IVCM yearly for 3 years after transplantation. Central subbasal nerve density was compared to a group of 20 healthy control subjects. With repeated examination of the transplant patients, care 
must be taken to scan approximately the same central corneal region to obtain an accurate representation of nerve regeneration.

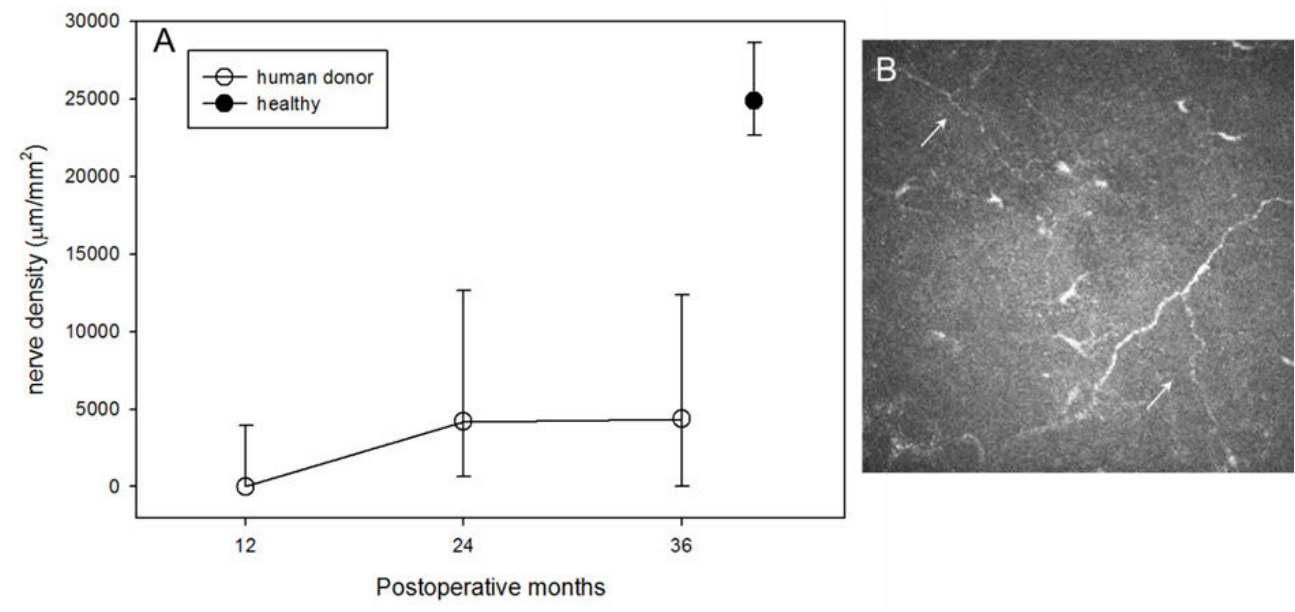

Figure 13. Recovery of central corneal subbasal nerve density after transplantation in a cohort of 9 patients. (A) A slow increase in subbasal nerve density is evident in the first postoperative years, however, density is significantly below the level in healthy corneas. (B) Image of regenerated nerves in the central cornea 3 years after transplantation, depicting thin, tortuous nerve branches (arrows) and a sparse presence of subbasal nerves.

In addition to longitudinal imaging of the central cornea, the peripheral cornea can provide insights into re-innervation of the transplanted cornea. The peripheral scar tissue that forms at the donor-to-recipient interface after surgery can serve as a clear landmark for repeated longitudinal imaging of the same microscopic field of view over time (Figure 14). Imaging the same microscopic field of view has been reported in both clinical [18], [20] and experimental animal studies [16], and provides a powerful demonstration of morphologic changes with time. In this example, imaging of an identical region in the cornea in 1 or 2 year intervals indicates changes in nerve presence and penetration into the implant, as well as longitudinal changes in the scar tissue itself.

\section{Cell quantification techniques}

In addition to nerves, corneal cells imaged by IVCM can also be subjected to quantitative analysis. The cells most often quantified are epithelial, stromal keratocytes, endothelium, and inflammatory. [10], [23], [24]

\subsection{Quantification of epithelial cell and keratocyte density}

A review of cell density in the various corneal layers reported by investigators using different types of confocal instruments is presented in Reference 10. With laser-scanning 

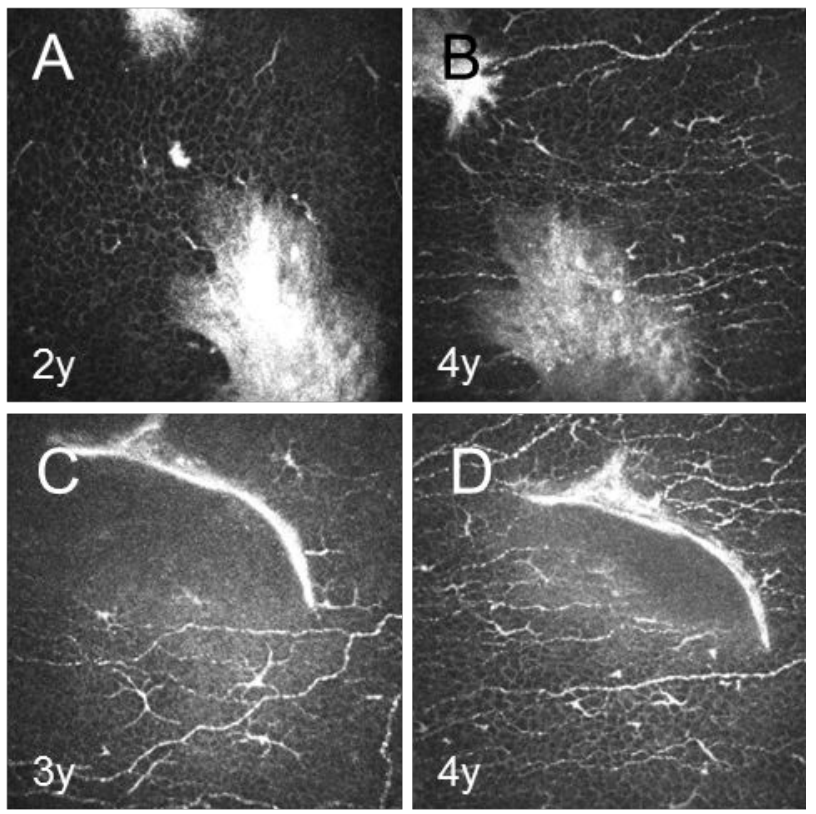

Figure 14. Longitudinal images of an identical region of peripheral cornea with scar tissue present between recipient and donor tissue. (A, B) In one patient, no subbasal nerves were observed in this peripheral region 2 years after transplantation. 4 years after transplantation, however, the identical region is infiltrated with a high density of nerves that traverse the scar tissue. In addition, light scatter from scar tissue has diminished significantly. (C, D) In another patient, the density of nerves in the immediate region of the scar increased significantly from 3 to 4 years postoperative. Note that the subbasal nerve paths are not constant but are constantly changing with time, a phenomenon that has been reported. [40]

confocal microscopy of the cornea, the fine axial resolution requires specification of the exact depth of the layer to be quantified. For example, in one study, epithelial cell and anterior stromal keratocyte density were determined longitudinally in patients with recurrent corneal erosions treated by laser ablation. [19] Epithelial cells were quantified at the superficial, wing, and basal cell layers, with wing cell images taken 20-25 $\mu \mathrm{m}$ below the corneal surface. Similarly, keratocytes were quantified at a distance of 10-15 $\mu \mathrm{m}$ below Bowman's layer. The resulting densities are specified as cells $/ \mathrm{mm}^{2}$. While some investigators prefer to report volumetric density (i.e., cells $/ \mathrm{mm}^{3}$ ), this notation is dependent upon the axial depth of field which varies between laser and non-laser confocal systems. Comparison between results obtained with laser confocal microscopes is possible by reporting density in cells per unit area.

Once depth is specified, the desired region of interest must be chosen, as it is often impractical to quantify all cells in an image, and desirable to exclude edge artifacts. Image processing techniques are then applied to the region of interest to enhance cell boundaries and facilitate manual cell counting. In Figure 15, a technique for image enhancement and manual cell density quantification is described. [19] 

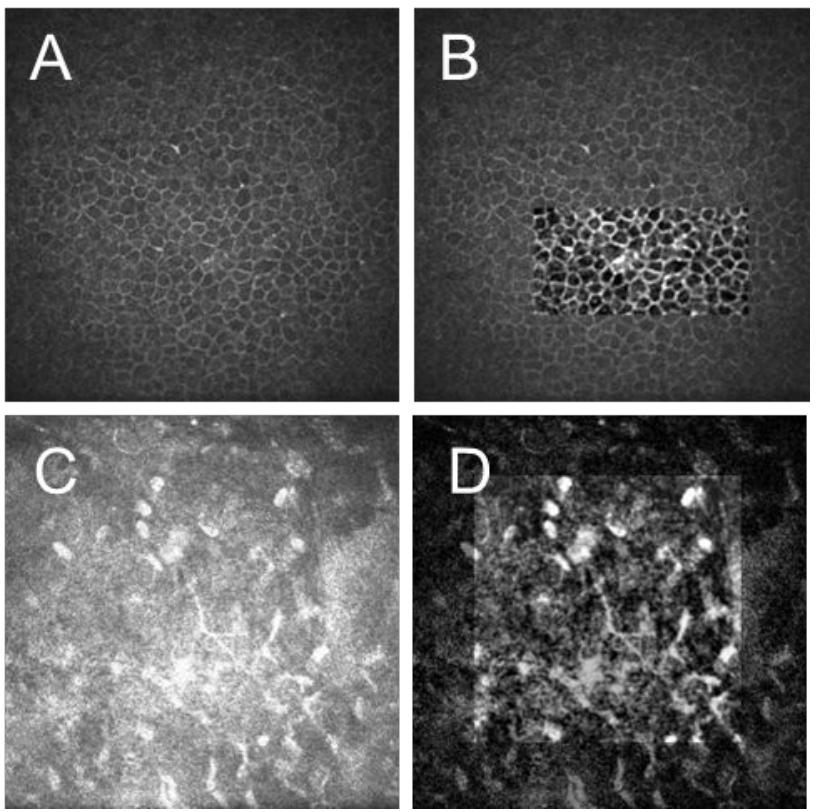

Figure 15. Example of cell quantification by IVCM in corneas affected by recurrent corneal erosions. (A) raw epithelial cell image. (B) image after selection of a $100 \times 200 \mu \mathrm{m}$ region of interest, which has been enhanced by bandpass filtering. Cells are then counted using point-and-click software, but excluding cells touching the bottom or left edge of the region, by convention (to avoid overestimation of density). Two observers perform the counting, and average cell count is converted to density by dividing by the area of the region of interest. The full technique is described in detail elsewhere. [19] (C) Image of keratocytes in the anterior stroma in a patient. (D) images after selection of a $250 \times 250$ $\mu \mathrm{m}$ region of interest, which has been enhanced by bandpass filtering. Cell quantification then proceeds in a similar manner as for epithelial cells.

\subsection{Quantification of dendritic cell density}

Inflammatory cells, in particular dendritic cells with long dendrites, migrate into the central cornea at the level of the subbasal nerve plexus in cases of inflammation. Several investigators have quantified the dendritic cell density in the normal and inflamed human cornea. [23], [24], [36] The threshold dendritic cell density in the normal central cornea is $34 \pm 3$ cells $/ \mathrm{mm}^{2}$, above which dendritic cell densities are pathologically elevated. [23] Contact lens wearers, for example, do not have inflamed corneas, but have an elevated central corneal dendritic cell density reported to be $78 \pm 25$ cells $/ \mathrm{mm}^{2}$. [48] Active inflammation was shown to correspond to central dendritic cell densities greater than $137 \pm 27$ cells $/ \mathrm{mm}^{2}$. [24] Quantification of dendritic cell density is achieved by manual counting of dendritic cells with dendrites in an IVCM field of view with the greatest number of visible dendritic cells in the central cornea. [36] The number of cells is then divided by the field of view $\left(400 \times 400 \mu \mathrm{m}\right.$, or $\left.0.16 \mathrm{~mm}^{2}\right)$ to yield density. An example of dendritic cell density quantification in patients with aniridia is given in Figure 16. Aniridia patients clearly have pathologically high dendritic cell density, however, only 2 of 11 subjects meet the criteria for having a clinically inflamed cornea. 

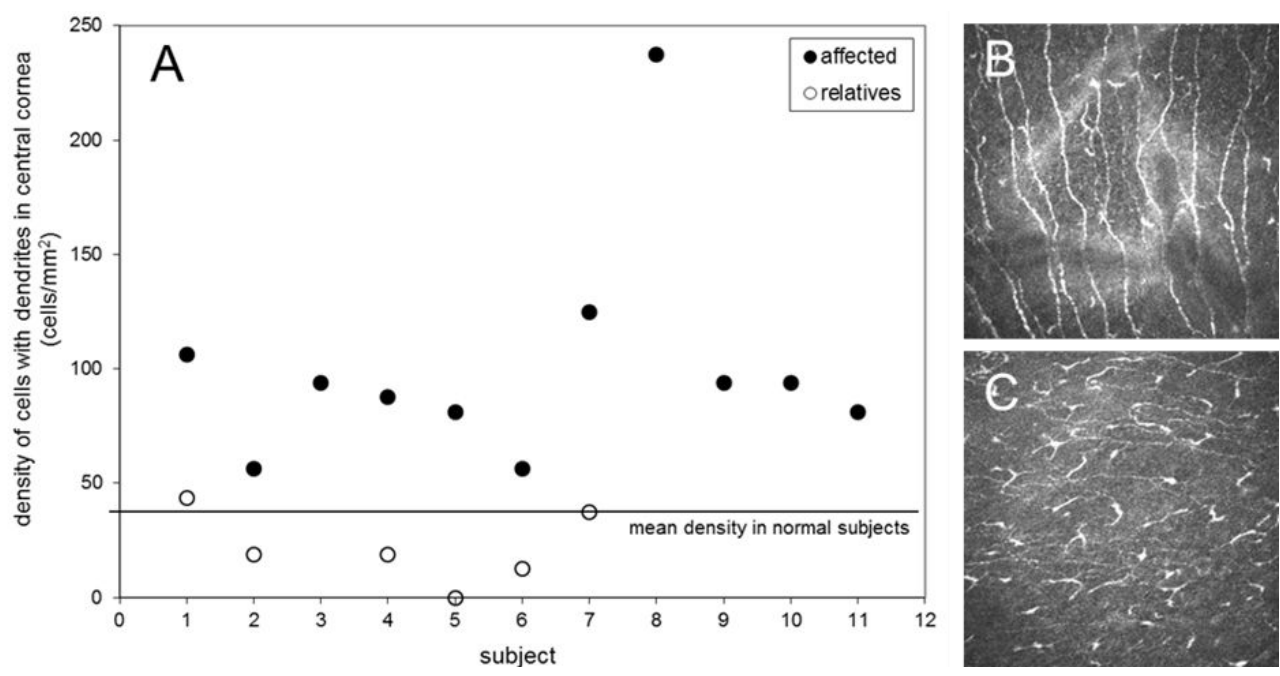

Figure 16. Quantification of mature dendritic cells in patients with aniridia. (A) dendritic cell density in aniridia patients (solid circles) is significantly elevated compared to unaffected relatives (open circles), who have a density considered to be normal (average normal density indicated by solid line, according to [23]). 2 of 11 aniridia subjects had cell density high enough to be considered clinically inflamed. (B) Image of the central subbasal plexus in an unaffected relative, with sparsely distributed mature dendritic cells, density 38 cells $/ \mathrm{mm}^{2}$. (C) Corresponding subbasal plexus in an aniridia patient, density 238 cells $/ \mathrm{mm}^{2}$.

\subsection{Fully automated cell counting technique}

Manual counting of epithelial cells and keratocytes can be time consuming and subject to observer interpretation and error. It is therefore desirable to automate the cell counting method. We have recently achieved fully-automated cell counting of epithelial wing cells and keratocytes from IVCM images taken from normal, healthy corneas. The automated technique is easy to implement and does not require expensive specialized software, but instead uses the free ImageJ platform. [49] In this method, one starts with the processed images depicted in Figure 15. Images are cropped to the region of interest, and thresholding is applied with an automatic (default) setting used to determine the threshold level. Alternatively, a fixed threshold level can be utilized for all images of a given cell type, based on prior experience of the optimum grayscale level for the particular type of images to be analyzed. Next, images should be inverted as required, such that cells appear as dark objects on a bright background. This is necessary for keratocytes, but not for wing cells as imaged in darkfield microscopy. The image is then converted into a binary representation (i.e., grayscale values are converted to either black or white), and a watershed function is used to automatically add boundaries where required, to depict a full separation between adjacent cells. Wing cells or keratocytes can then be automatically counted using the 'analyze particles' function in ImageJ. Because the function does not allow inclusion of cells touching only two of the four boundaries, the function is applied twice (once including and once excluding all four boundaries) and the cell count values are averaged. An example of this procedure is given in Figure 17. 

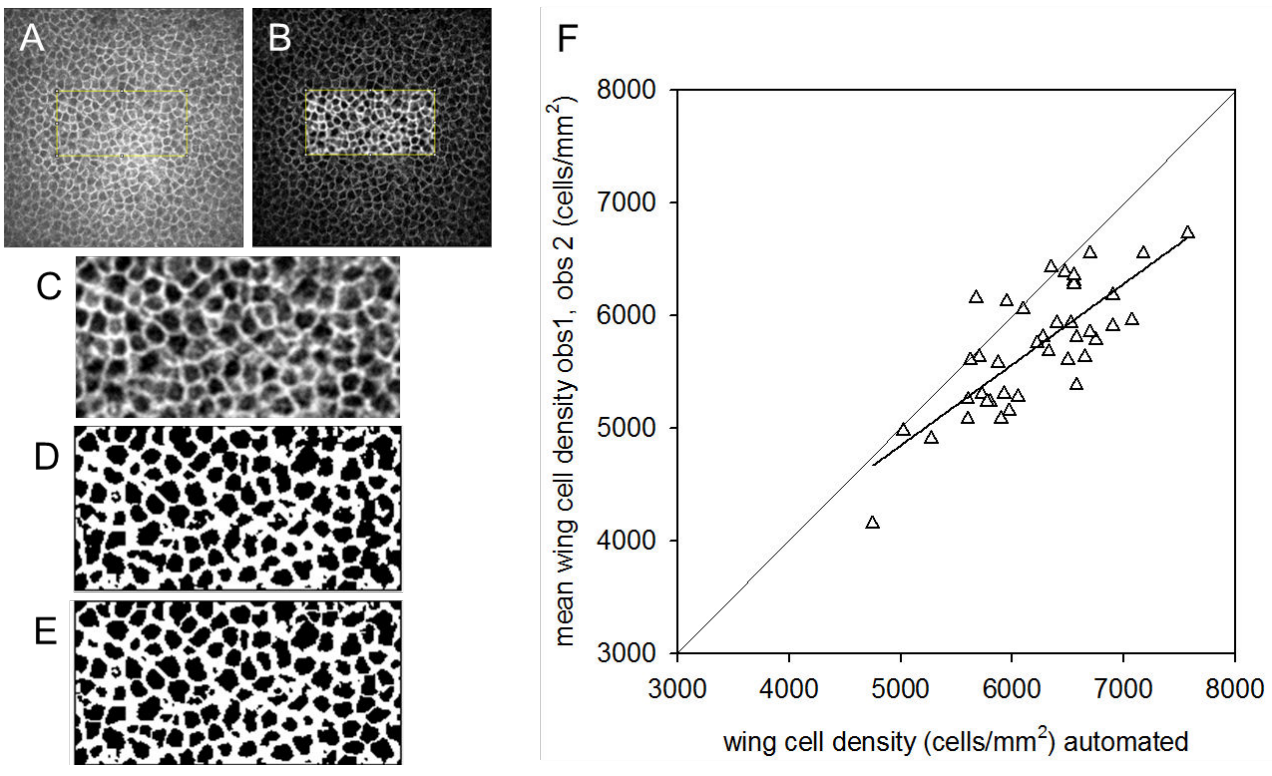

Figure 17. Fully automated analysis of wing cell density from IVCM images. (A) Wing cell layer from a healthy human cornea. (B) Bandpass-filtered region of interest. (C) Cropped, magnified view of region of intrest with automatic thresholding applied. (D) Binary-converted image. (E) Watershed function applied to the binary image to delineate separate cells. Note that the subsequent automatic cell counting is configured to ignore dark areas smaller than a given size in square pixels. This avoids the small point-like artifacts in the image from being included as cells. (F) It is always important to compare the agreement of a new automated method with the original manual cell counting method, to determine the degree of agreement between methods and isolate any source of bias. Here, the automated technique appears to yield slightly larger values for cell density than the manual method (averaged value of two observers).

\section{Improved axial resolution and 3D rendering}

\subsection{Technical requirements}

Although the axial resolution of IVCM is approximately $4 \mu \mathrm{m}$, situations arise where a finer resolution or depth spacing of images is required. This is of particular importance when analyzing small structures or attempting to create 3D models or high-resolution cross-sections for in vivo histology-type analysis. These requirements can be addressed by combined hardware and software-analysis approaches. In terms of hardware, the HRT3-RCM system

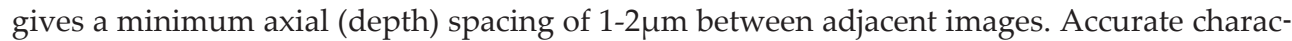
terization of structures as thin as Bowman's layer (approximately $10 \mu \mathrm{m}$ thick in humans) is therefore difficult. [50] An attachment to the HRT3-RCM system, however, can be used to achieve a finer depth spacing of images. The attachment enables fine motorized adjustment of 
the microscope objective in the axial direction, to achieve images with axial spacing as fine as $0.5 \mu \mathrm{m} .{ }^{4}$ Analysis of images can be manual or software-assisted. In the following example, software-assisted analysis is used to produce high-resolution histologic-type sections from live tissue and 3D views of lymphatic vessels trafficking inflammatory cells in the rat cornea.

\subsection{High-resolution $3 \mathrm{D}$ in vivo imaging of corneal lymphatics}

Corneal lymphatics are not normally present in the cornea, however, they can invade the corneal stroma during inflammation. [17], [51] In a rat model of inflammatory angiogenesis, the first corneal lymph vessels are observed from 7 to 14 days after the induction of inflammation by placement of a nylon suture through the corneal stroma. [17] Lymphatic vessels are normally analyzed ex-vivo by histologic staining in tissue sections to reveal details of the lymphatic vessel lumen in cross-section [52], however, IVCM enables the histologic crosssection to be analyzed in vivo without removing tissue from the animal. To achieve this, confocal image stacks containing lymph vessels are first obtained using the motor-driven axial positioning attachment. [4] Setting the acquisition rate to 30 frames/sec, a stack of images is obtained during a depth scan of $30 \mu \mathrm{m}$ in the axial direction. The stack consists of 60 confocal image frames, with an axial spacing of $0.5 \mu \mathrm{m}$ between images. Using 3D tools from ImageJ, any desired cross-section in the $\mathrm{xz}$ or $\mathrm{yz}$ directions ( $\mathrm{z}$ is the axial direction) can be obtained for analysis (Figure 18). An image stack can also serve as input for 3D rendering software, for example the Volocity Visualization software (Volocity 6.0, Perkin Elmer Inc., Waltham, MA, USA). Motion artifacts in the stack can be auto-corrected using an image alignment tool in Volocity, resulting in smoother boundaries when creating a 3D model. With the model, structures can be studied from any desired viewpoint (Figure 18).

\section{Future perspective - Correlative microscopy}

Although IVCM provides unparalleled possibilities for microscopic in vivo investigation of the cornea, one of the principal drawbacks of the technique is that it provides only morphologic information. The composition and identity of structures and cells viewed by IVCM can only be determined by tissue excision and immunohistological methods. In many cases, however, tissue sampling or excision is impractical or unethical, particularly in human subjects where non-destructive imaging is required.

In the future, correlative microscopy techniques will be required to overcome this limit. With this approach, the same tissue is first examined in vivo, then after tissue excision (in animal models, or after transplantation in humans, for example) in stained sections or by light, fluorescence, or electron microscopy. Comparison of the same structures in vivo and ex vivo by this approach yields insights into the correlation between in vivo morphology and identity and composition of the structures. An example of corneal tissue examined by IVCM prior to transplantation and further analysis by light microscopy and transmission electron microscopy is given in Figure 19. Such analysis improves the ability to interpret IVCM images and recognize pathologic features in vivo. 

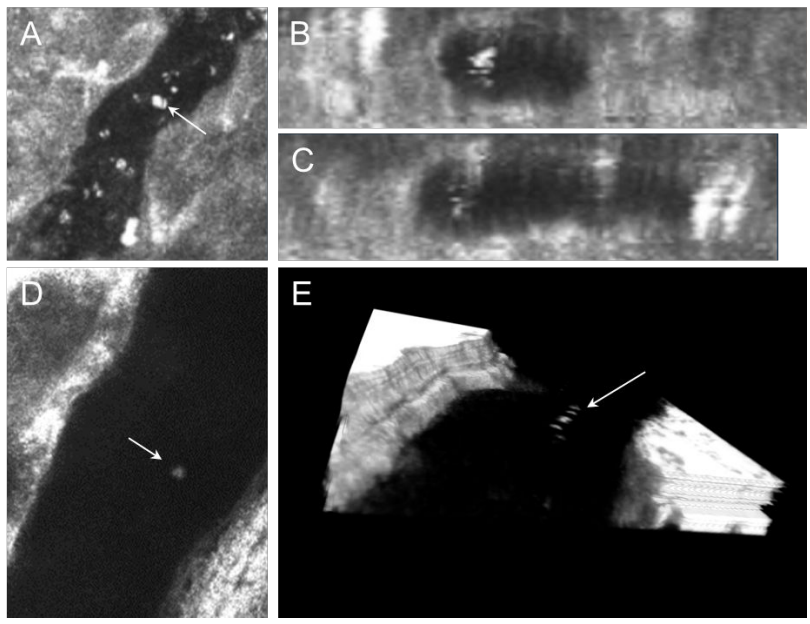

Figure 18. High-resolution 3D imaging of corneal lymphatics in the rat, using IVCM. (A) A standard xy confocal section depicting a lymphatic in the rat cornea during sustained inflammation, with leukocyte traffic visible within the lymph vessel (arrow). (B) An image stack of the same lymph vessel viewed in the xz-plane provides a live histological-type view of the lymph vessel cross-section, with a cell visible in the lymph fluid. (C) yz-plane of the same lymph vessel. (D) A larger, limbal lymph vessel in the rat contains a flowing leukocyte. (E) A confocal image stack obtained during the flow of the leukocyte is rendered into a 3D model, enabling the cell trajectory within the lymphatic lumen to be visualized (arrow).

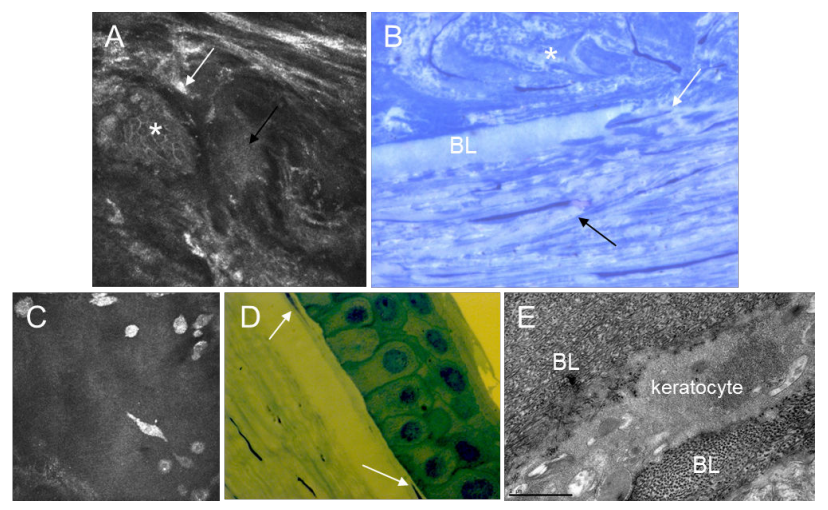

Figure 19. Example of correlating confocal microscopy findings with light and electron microscopy in the same tissue sample. (A) Pre-transplantation IVCM image from a 35y old female with lattice dystrophy, depicting disrupted Bowman's layer (black arrow) along with a presumed keratocyte (white arrow) and basal epithelial cells (asterisk) present at the same corneal depth. Note the uneven light scattering from the stroma, indicating disrupted collagen organization. (B) Light microscope image of a section of the same cornea as in (A) after resection, indicating the histologic appearance of a truncated Bowman's layer (BL), presumed keratocyte at the same depth as BL (white arrow), and disruptions in the stromal collagen (black arrow). (C) Pre-transplantation IVCM image from a 61y old female with endothelial dystrophy, with keratocytes present in the normally acellular BL.(D) Light microscopy section of the same cornea after resection, with keratocytes clearly visible in or anterior to BL. (E) Confirmation by electron microscopy that a keratocyte is present within the non-lamellar collagen of BL. 
An example of the use of multiple imaging modalities to confirm diagnosis is given in Figure 20. In this case, upon routine examination at the optometrist an asymptomatic $35 \mathrm{y}$ old female was found to have fine, refractile structures distributed throughout both corneas. Slit-lamp biomicroscopy revealed golden-brown colored deposits, which by optical coherence tomography were highly light-scattering and determined to be confined to the anterior one-third of the stroma, which appeared otherwise normal. IVCM confirmed the anterior stromal location of a dense population of light-scattering crystalline structures which were not co-located with keratocyte nuclei. IVCM confirmed the presence of the structures peripherally, and a peripheral biopsy was taken. The biopsy sample was analyzed with transmission electron microscopy, where the crystalline structures could be localized to the keratocyte cytoplasm, confirming the diagnosis of non-nephropathic adult cystinosis.
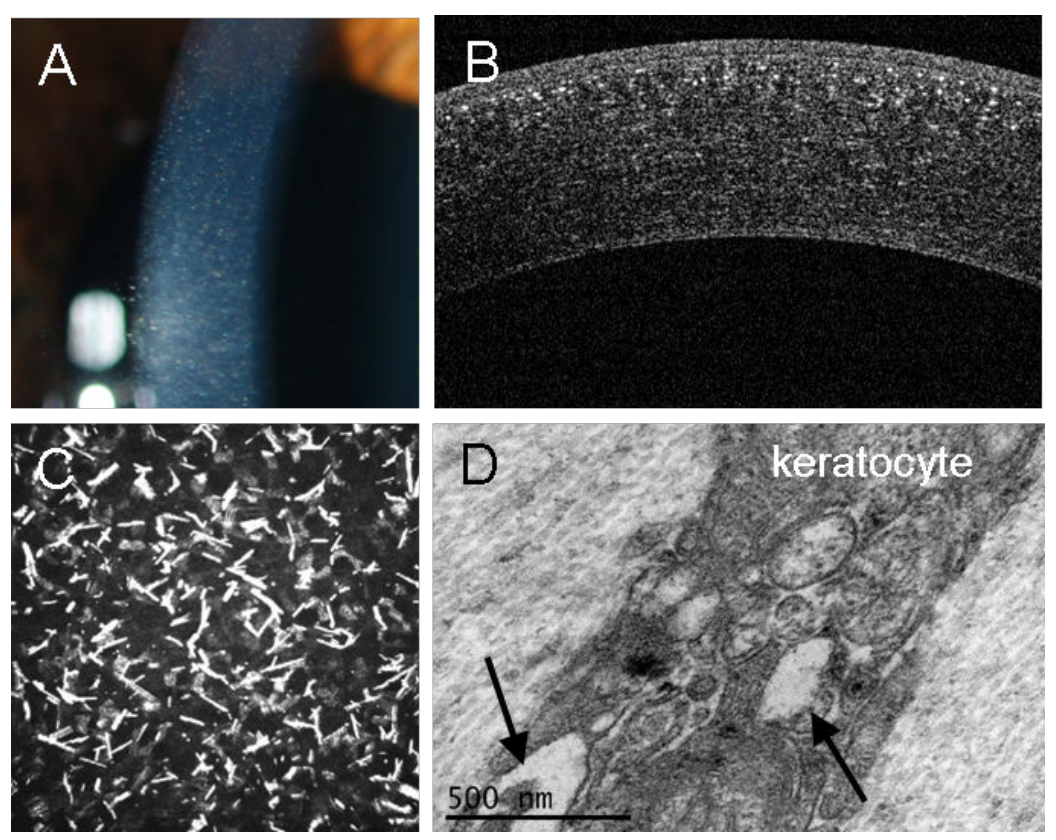

Figure 20. Example of multiple imaging techniques used to characterize and diagnose non-nephropathic adult cystinosis in the cornea of a 35y old female. (A) Slit lamp image of fine, golden refractile particles distributed throughout the cornea. (B) Fourier-domain optical coherence tomography image of the cornea indicating distribution of lightscattering objects in the anterior one-third of the cornea. (C) In vivo confocal microscopy in the anterior cornea indicates linear, crystalline, highly scattering structures in the anterior stroma, located outside the keratocyte nucleus. (D) Transmission electron microscopy image from a biopsy sample taken from the same patient, indicating the presence of cysteine crystals within the cytoplasm of the keratocytes (arrows).

An example of exact correlation between in vivo and immunologically stained tissue is given in Figure 21. In a rat model of angiogenesis, blood vessels and inflammatory cells were observed first by IVCM, by the technique depicted in Figure 2B. Following live imaging, the cornea was excised at specific time points ( 2 and 7 days after induction of inflammation in this 
example). The tissue was then prepared as a whole-mount corneal sample, immunologically labeled with primary antibodies against specific cellular and vascular structures, and imaged in the same en face view as the IVCM provides, but instead using a high-resolution laserscanning confocal fluorescence microscope to detect fluorescent signatures of the secondary antibodies. The whole-mount sample is then manually translated during scanning to locate the same vessel structures observed in vivo. This technique requires access to the pre-saved IVCM images while performing the fluorescence microscopy, and additionally requires distinct vessel configurations that serve as landmarks to aid in the localization of the same structures in fluorescence. When the exact region is found in fluorescence, comparison with the in vivo images can yield a wealth of information about the structures viewed in vivo. For example, as shown in Figure 21, the technique has confirmed pericyte coverage of certain pathologic blood vessels, and has resulted in the first observations of corneal lymphatics in a live animal cornea without labeling. [17]
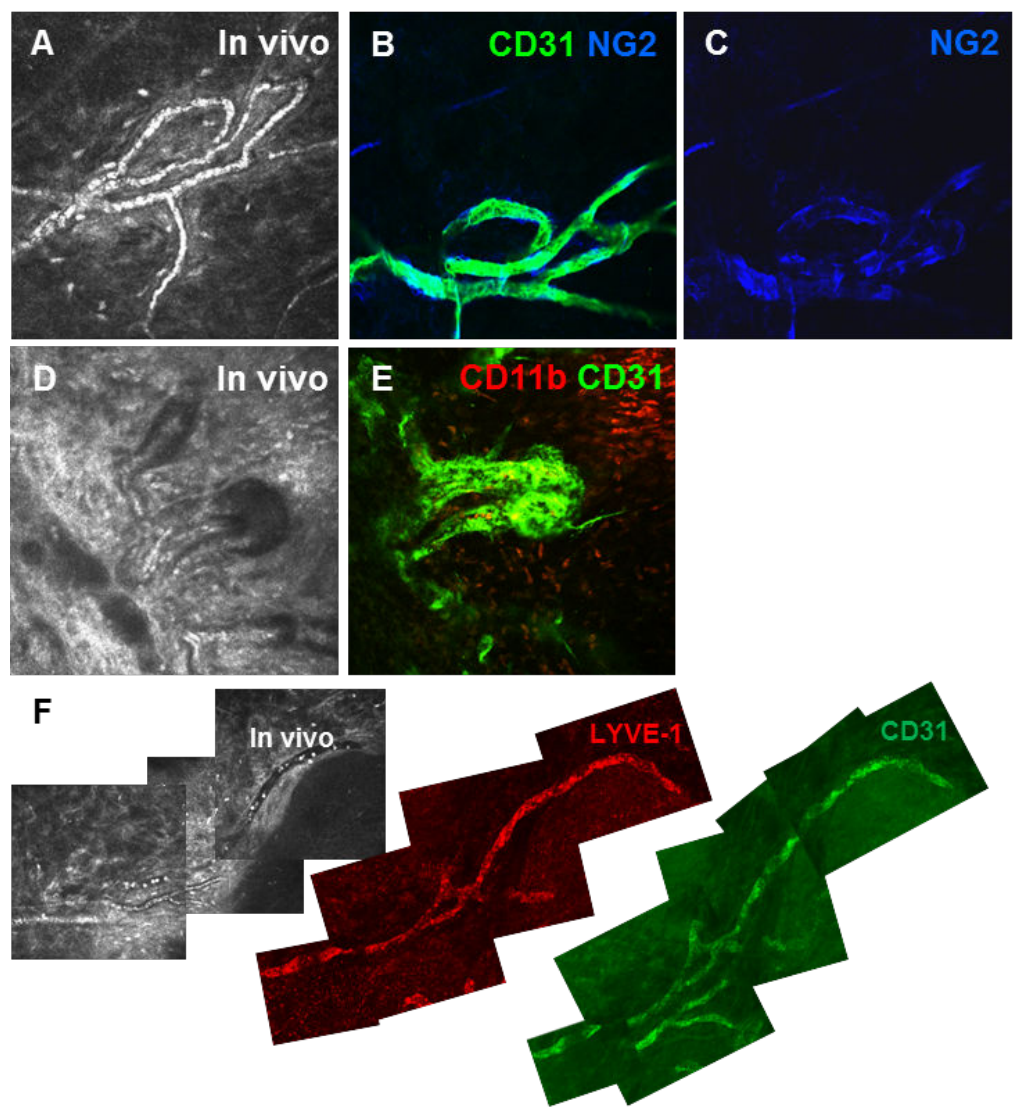

Figure 21. Examples of exact correlative in vivo/ex vivo microscopy. (A-C) The same pathologic corneal blood vessels viewed in vivo and ex vivo in an en face orientation, after immunofluorescent staining for blood vessel vascular endo- 
thelium (CD31) and pericytes/mural cells (NG2). The images indicate the appearance of mature, pericyte-covered blood vessels in the cornea that are more resistant to regression. (D, E) In vivo and immunofluorescent view of a highly dilated limbal blood vessel prior to angiogenic sprouting. The blood vessel is CD31+ while cells inside and around the vessel are CD11 b+ inflammatory myeloid cells. The images indicate the inflammatory cells do not physically incorporate into the vascular endothelium during the budding process, but likely serve a paracrine role. (F) Confirmation of the in vivo appearance of lymphatic vessels in the rat cornea, which have a different morphology than that of blood vessels in vivo. Localization of the same lymph vessel after tissue whole mounting and immunostaining for lymphatic endothelial marker LYVE-1 (which blood vessels do not express) served to confirm the lymphatic nature of the in vivo vessel structure.

\section{Conclusion}

Besides the direct clinical and preclinical application of IVCM to the cornea - which in itself yields a wealth of information pertaining to corneal anatomy, physiology, and pathology - the technique can serve as an instructional tool in the use of laser-scanning confocal microscopy. Many of the acquisition, processing, and analysis techniques described in this chapter are applicable to other confocal microscopes, different tissue types, and other fields of research and practice. Moreover, IVCM of the cornea is a constantly growing and evolving field, and it is envisaged that the technique will extend our knowledge of not only the eye, but of basic biologic and physiologic processes occurring in other parts of the body. Finally it is hoped that other fields of scientific endeavor will not only benefit from knowledge gained from IVCM of the cornea, but, conversely, that knowledge obtained from laser-scanning confocal microscopy in other fields can be applied to the cornea.

\section{Acknowledgements}

The authors wish to acknowledge kind contributions to the work presented from the following individuals: Tor Paaske Utheim and Xiangjun Chen, Oslo University Department of Ophthalmology, Catharina Traneus-Röckert, Department of Pathology, Linköping University, Thu Ba Wihlmark and Marina Koulikovska, Department of Ophthalmology, Linköping University Hospital, Joachim Stave, Department of Ophthalmology, University of Rostock, and Ulf Stenevi and Charles Hanson, Sahlgrenska University Hospital, Gothenburg. The authors also wish to acknowledge financial support from the King Gustav V and Queen Margaretas Freemasons Foundation and the Carmen and Bertil Regnérs Foundation.

\section{Author details}

Neil Lagali*, Beatrice Bourghardt Peebo, Johan Germundsson, Ulla Edén, Reza Danyali, Marcus Rinaldo and Per Fagerholm

Department of Clinical and Experimental Medicine, Faculty of Health Sciences, Linköping University, Linköping, Sweden 


\section{References}

[1] C. Stephen Foster, Dimitri T Azar, Claes H Dohlman, Eds. Smolin and Thoft's The Cornea: Scientific Foundations and Clinical Practice, 4th Edition, Lippincott Williams Wilkins 2004.

[2] Oliveira-soto, L, \& Efron, N. Assessing the cornea by in vivo confocal microscopy. Clin Experiment Ophthalmol (2003). , 31, 83-84.

[3] Atlas of Confocal Laser Scanning In-vivo Microscopy in OphthalmologyBy R.F. Guthoff, C. Baudouin, J. Stave,. Springer, Berlin (2006).

[4] Rostock Cornea Module Z-motor drive Unit. Joachim Stave (Rostock). Personal communication.

[5] Niederer, R. L, \& Mcghee, C. N. Clinical in vivo confocal microscopy of the human cornea in health and disease. Prog Retin Eye Res. (2010). , 29, 30-58.

[6] Mclaren, J. W, Nau, C. B, Kitzmann, A. S, \& Bourne, W. M. Keratocyte density: comparison of two confocal microscopes. Eye Contact Lens. (2005). , 31, 28-33.

[7] Lagali, N, Griffith, M, Fagerholm, P, Merrett, K, Huynh, M, \& Munger, R. Innervation of tissue-engineered recombinant human collagen-based corneal substitutes: a comparative in-vivo confocal microscopy study. Invest Ophthalmol Vis Sci, (2008). , $49,3895-3902$.

[8] Guthoff, R. F, Zhivov, A, \& Stachs, O. In vivo confocal microscopy, an inner vision of the cornea- a major review. Clin Experiment Ophthalmol. (2009). Jan;, 37(1), 100-17.

[9] Erie, J. C, Mclaren, J. W, \& Patel, S. V. Confocal microscopy in ophthalmology. Am J Ophthalmol. (2009). , 148, 639-46.

[10] Patel, D. V, \& Mcghee, C. N. Contemporary in vivo confocal microscopy of the living human cornea using white light and laser scanning techniques: a major review. Clin Experiment Ophthalmol. (2007). , 35, 71-88.

[11] Labbé, A, Liang, H, Martin, C, Brignole-baudouin, F, Warnet, J. M, \& Baudouin, C. Comparative anatomy of laboratory animal corneas with a new-generation high-resolution in vivo confocal microscope. Curr Eye Res. (2006). , 31, 501-9.

[12] Lagali, N, Griffith, M, Shinozaki, N, Fagerholm, P, \& Munger, R. Innervation of tissue-engineered corneal implants in a porcine model: a 1-year in-vivo confocal microscopy study. Invest Ophthalmol Vis Sci (2007). , 48, 3537-3544.

[13] Hackett, J, Lagali, N, Merrett, K, Edelhauser, H, Sun, Y, Gan, L, Griffith, M, \& Fagerholm, P. Biosynthetic corneal implants for replacement of pathologic corneal tissue: performance in a controlled rabbit alkali burn model. Invest Ophthalmol Vis Sci (2011). , 52, 651-657. 
[14] Hovakimyan, M, Guthoff, R, Reichard, M, Wree, A, Nolte, I, \& Stachs, O. In vivo confocal laser-scanning microscopy to characterize wound repair in rabbit corneas after collagen cross-linking. Clin Experiment Ophthalmol. (2011). , 39, 899-909.

[15] Liang, H, Brignole-baudouin, F, Labbé, A, Pauly, A, Warnet, J. M, \& Baudouin, C. LPS-stimulated inflammation and apoptosis in corneal injury models. Mol Vis. (2007). , 13, 1169-80.

[16] Peebo, B. B, Fagerholm, P, Traneus-röckert, C, \& Lagali, N. Time-lapse in vivo imaging of corneal angiogenesis: the role of inflammatory cells in capillary sprouting. Invest Ophthalmol Vis Sci (2011). , 52, 3060-8.

[17] Peebo, B. B, Fagerholm, P, Traneus-röckert, C, \& Lagali, N. Cellular-level characterization of lymph vessels in live, unlabelled corneas by in-vivo confocal microscopy. Invest Ophthalmol Vis Sci (2010). , 51, 830-835.

[18] Bourghardt Peebo BFagerholm P, Lagali N. Transient anterior corneal deposits in a human immunodeficiency virus-positive patient. Cornea (2010). , 29, 1323-1327.

[19] Lagali, N, Germundsson, J, \& Fagerholm, P. The role of Bowman's layer in anterior corneal regeneration after shallow-depth phototherapeutic keratectomy: a prospective, morphological study using in-vivo confocal microscopy. Invest Ophthalmol Vis Sci (2009). , 50, 4192-4198.

[20] Peebo, B. B, Fagerholm, P, Traneus-röckert, C, \& Lagali, N. Cellular level characterization of capillary regression in inflammatory angiogenesis using an in vivo corneal model. Angiogenesis (2011). , 14, 393-405.

[21] Knappe, S, Stachs, O, Zhivov, A, Hovakimyan, M, \& Guthoff, R. Results of confocal microscopy examinations after collagen cross-linking with riboflavin and UVA light in patients with progressive keratoconus. Ophthalmologica. (2011). , 225, 95-104.

[22] Erie, J. C, Hodge, D. O, \& Bourne, W. M. Confocal microscopy evaluation of stromal ablation depth after myopic laser in situ keratomileusis and photorefractive keratectomy. J Cataract Refract Surg. (2004). , 30, 321-5.

[23] Zhivov, A, Stave, J, Vollmar, B, \& Guthoff, R. In vivo confocal microscopic evaluation of Langerhans cell density and distribution in the normal human corneal epithelium. Graefes Arch Clin Exp Ophthalmol. (2005). , 243, 1056-61.

[24] Mastropasqua, L, Nubile, M, Lanzini, M, Carpineto, P, Ciancaglini, M, \& Pannellini, T. Di Nicola M, Dua HS. Epithelial dendritic cell distribution in normal and inflamed human cornea: in vivo confocal microscopy study. Am J Ophthalmol. (2006). , 142, 736-44.

[25] Patel, D. V. McGhee CNJ. Acanthamoeba keratitis: a comprehensive photographic reference of common and uncommon signs. Clin Experiment Ophthalmol (2009). , 37, 232-238. 
[26] Vaddavalli, P. K, Garg, P, Sharma, S, Sangwan, V. S, Rao, G. N, \& Thomas, R. Role of confocal microscopy in the diagnosis of fungal and acanthamoeba keratitis. Ophthalmology. (2011). , 118, 29-35.

[27] Hau, S. C, Dart, J. K, Vesaluoma, M, Parmar, D. N, Claerhout, I, Bibi, K, \& Larkin, D. F. Diagnostic accuracy of microbial keratitis with in vivo scanning laser confocal microscopy. Br J Ophthalmol (2010). , 94, 982-7.

[28] Shiraishi, A, Uno, T, Oka, N, Hara, Y, Yamaguchi, M, \& Ohashi, Y. In vivo and in vitro laser confocal microscopy to diagnose acanthamoeba keratitis. Cornea (2010). , $29,861-5$.

[29] Niederer, R. L, Perumal, D, Sherwin, T, \& Mcghee, C. N. Age-related differences in the normal human cornea: a laser scanning in vivo confocal microscopy study. Br J Ophthalmol (2007). , 91, 1165-9.

[30] Patel, D. V, Sherwin, T, \& Mcghee, C. N. Laser scanning in vivo confocal microscopy of the normal human corneoscleral limbus. Invest Ophthalmol Vis Sci. (2006). , 47, 2823-7.

[31] Shortt, A. J, Secker, G. A, Munro, P. M, Khaw, P. T, Tuft, S. J, \& Daniels, J. T. Characterization of the limbal epithelial stem cell niche: novel imaging techniques permit in vivo observation and targeted biopsy of limbal epithelial stem cells. Stem Cells. (2007). , 25, 1402-9.

[32] Takahashi, N, Chikama, T, Yanai, R, \& Nishida, T. Structures of the corneal limbus detected by laser-scanning confocal biomicroscopy as related to the palisades of Vogt detected by slit-lamp microscopy. Jpn J Ophthalmol. (2009). , 53, 199-203.

[33] Miri, A, Al-aqaba, M, Otri, A. M, Fares, U, Said, D. G, Faraj, L. A, \& Dua, H. S. In vivo confocal microscopic features of normal limbus. Br J Ophthalmol. (2012). , 96, 530-6.

[34] Deng, S. X, Sejpal, K. D, Tang, Q, Aldave, A. J, Lee, O. L, \& Yu, F. Characterization of limbal stem cell deficiency by in vivo laser scanning confocal microscopy: a microstructural approach. Arch Ophthalmol. (2012). , 130, 440-5.

[35] Hong, J, Zheng, T, Xu, J, Deng, S. X, Chen, L, Sun, X, Le, Q, \& Li, Y. Assessment of limbus and central cornea in patients with keratolimbal allograft transplantation using in vivo laser scanning confocal microscopy: an observational study. Graefes Arch Clin Exp Ophthalmol. (2011). , 249, 701-8.

[36] Edén, U, Fagerholm, P, Danyali, R, \& Lagali, N. Pathologic epithelial and anterior corneal nerve morphology in early-stage congenital aniridic keratopathy. Ophthalmology. (2012). , 119, 1803-10.

[37] Müller, L. J, Marfurt, C. F, Kruse, F, \& Tervo, T. M. Corneal nerves: structure, contents and function. Exp Eye Res. (2003). , 76, 521-42. 
[38] Patel, D. V, \& Mcghee, C. N. In vivo confocal microscopy of human corneal nerves in health, in ocular and systemic disease, and following corneal surgery: a review. Br J Ophthalmol. (2009). , 93, 853-60.

[39] Patel, D. V, \& Mcghee, C. N. Mapping of the normal human corneal sub-Basal nerve plexus by in vivo laser scanning confocal microscopy. Invest Ophthalmol Vis Sci. (2005). , 46, 4485-8.

[40] Patel, D. V, \& Mcghee, C. N. In vivo laser scanning confocal microscopy confirms that the human corneal sub-basal nerve plexus is a highly dynamic structure. Invest Ophthalmol Vis Sci. (2008). , 49, 3409-12.

[41] Patel, D. V, \& Mcghee, C. N. Mapping the corneal sub-basal nerve plexus in keratoconus by in vivo laser scanning confocal microscopy. Invest Ophthalmol Vis Sci. (2006). , 47, 1348-51.

[42] Zhivov, A, Blum, M, Guthoff, R, \& Stachs, O. Real-time mapping of the subepithelial nerve plexus by in vivo confocal laser scanning microscopy. Br J Ophthalmol. (2010). , 94, 1133-5.

[43] Turuwhenua, J. T, Patel, D. V, \& Mcghee, C. N. Fully automated montaging of laser scanning in vivo confocal microscopy images of the human corneal subbasal nerve plexus. Invest Ophthalmol Vis Sci. (2012). , 53, 2235-42.

[44] Szaflik, J. P. Comparison of in vivo confocal microscopy of human cornea by white light scanning slit and laser scanning systems. Cornea. (2007). , 26, 438-45.

[45] Marfurt, C. F, Cox, J, Deek, S, \& Dvorscak, L. Anatomy of the human corneal innervation. Exp Eye Res. (2010). , 90, 478-92.

[46] Al-aqaba, M. A, Fares, U, Suleman, H, Lowe, J, \& Dua, H. S. Architecture and distribution of human corneal nerves. Br J Ophthalmol. (2010). , 94, 784-9.

[47] Waring, G. O. rd,. Rodrigues MM, Laibson PR.Corneal dystrophies. I. Dystrophies of the epithelium, Bowman's layer and stroma. Surv Ophthalmol. (1978). , 23, 71-122.

[48] Zhivov, A, Stave, J, Vollmar, B, \& Guthoff, R. In vivo confocal microscopic evaluation of langerhans cell density and distribution in the corneal epithelium of healthy volunteers and contact lens wearers. Cornea. (2007). , 26, 47-54.

[49] Rasband, W.S, \& Image, . , U. S. National Institutes of Health, Bethesda, Maryland, USA, http://imagej.nih.gov/ij/, 1997-2012.

[50] Germundsson, J, Fagerholm, P, Koulikovska, M, \& Lagali, N. S. An accurate method to determine Bowman's layer thickness in vivo in the human cornea. Invest Ophthalmol Vis Sci. (2012). , 53, 2354-9.

[51] Regenfuss, B, Bock, F, Parthasarathy, A, \& Cursiefen, C. Corneal (lymph)angiogenesis--from bedside to bench and back: a tribute to Judah Folkman. Lymphat Res Biol. (2008). 
[52] Cursiefen, C, Schlötzer-schrehardt, U, Küchle, M, Sorokin, L, Breiteneder-geleff, S, Alitalo, K, \& Jackson, D. Lymphatic vessels in vascularized human corneas: immunohistochemical investigation using LYVE-1 and podoplanin. Invest Ophthalmol Vis Sci. (2002). , 43, 2127-35. 